\title{
On Grüss inequalities within generalized K-fractional integrals
}

\author{
Saima Rashid', Fahd Jarad², Muhammad Aslam Noor ${ }^{3}$, Khalida Inayat Noor ${ }^{3}$, Dumitru Baleanu ${ }^{2,45^{*}}$ and
} Jia-Bao Liu' ${ }^{6,7}$

\section{"Correspondence:}

dumitru@cankaya.edu.tr

${ }^{2}$ Department of Mathematics,

Cankaya University, Ankara, Turkey

${ }^{4}$ Institute of Space Sciences,

Magurele-Bucharest, Romania

Full list of author information is

available at the end of the article

\begin{abstract}
In this paper, we introduce the generalized $\mathcal{K}$-fractional integral in the frame of a new parameter $\mathcal{K}>0$. This paper offers some new important inequalities of Grüss type using the generalized $\mathcal{K}$-fractional integral and associated integral inequalities. Our results with this new integral operator have the abilities to be implemented for the evaluation of many mathematical problems related to the real world applications.
\end{abstract}

MSC: 26E60; 26D15; 26D10; 26A33

Keywords: Grüss inequality; Generalized $\mathcal{K}$-fractional integral; Integral inequality

\section{Introduction}

There are numerous problems wherein fractional derivatives (non-integer order derivatives and integrals) attain a valuable position [1-9]. It must be emphasized that fractional derivatives are furnished in many techniques, especially, characterizing three distinct approaches, which we are able to mention in an effort to grow the work in certainly one of them. Every classical fractional operator is typically described in terms of a particular significance. There are many well-recognized definitions of fractional operators, we can point out the Riemann-Liouville, Caputo, Grunwald-Letnikov, and Hadamard operators [10], whose formulations include integrals with singular kernels and which may be used to check the issues involving the reminiscence effect [11]. Some special new formulas for the fractional operators can be found in the literature [12]. These new formulas are different from the classical formulas in numerous components. As an example, classical fractional derivatives are described in such a manner that in the limit wherein the order of the derivative is an integer, one recovers the classical derivatives in the sense of Newton and Leibniz. There have additionally been currently proposed new fractional operators [13] with a corresponding integral whose kernel may be a non-singular mapping, as an instance, a Mittag-Leffler function. Additionally, in such instances, integer-order derivatives are rediscovered by supposing suitable limits for the values of their parameters.

On the other hand, there are numerous approaches to acquiring a generalization of classical fractional integrals. Many authors introduced new fractional operators generated from general classical local derivatives (see [14-17] and the references therein). Other authors introduced a parameter and enunciated a generalization for fractional integrals on

(c) The Author(s) 2020. This article is licensed under a Creative Commons Attribution 4.0 International License, which permits use, sharing, adaptation, distribution and reproduction in any medium or format, as long as you give appropriate credit to the original author(s) and the source, provide a link to the Creative Commons licence, and indicate if changes were made. The images or other third party material in this article are included in the article's Creative Commons licence, unless indicated otherwise in a credit line to the material. If material is not included in the article's Creative Commons licence and your intended use is not permitted by statutory regulation or exceeds the permitted use, you will need to obtain permission directly from the copyright holder. To view a copy of this licence, visit http://creativecommons.org/licenses/by/4.0/. 
a selected space. These are called generalized $\mathcal{K}$-fractional integrals. For such operators, we refer to $[4,18-20]$ and the works cited in them.

It is well known that inequalities have potential applications in the technology, scientific studies, and analysis [21-32] and numerous mathematical problems such as approximation theory, statistical analysis, and human social sciences [33-59]. In perspective on the more extensive applications, such variants have acquired large interest. Presently, authors have provided the unique version of such inequalities, which may be beneficial in the investigation of diverse forms of integrodifferential and difference equations. Those variants are an extensive instrument to take a gander at the classes of differential and integral equations.

The most celebrated Grüss inequality can be described as follows.

Theorem 1.1 (see [60]) Let $\mathcal{R}$ be a set of real numbers, $m, \mathcal{M}, n, \mathcal{N} \in \mathcal{R}$ and $\mathcal{P}, \mathcal{S}$ : $\left[\sigma_{1}, \sigma_{2}\right] \rightarrow \mathcal{R}$ be two positive functions such that $m \leq \mathcal{P}\left(\mathfrak{d}_{1}\right) \leq \mathcal{M}$ and $n \leq \mathcal{S}\left(\mathfrak{d}_{1}\right) \leq \mathcal{N}$ for all $\mathfrak{d}_{1} \in\left[\sigma_{1}, \sigma_{2}\right]$. Then

$$
\begin{aligned}
& \left|\frac{1}{\sigma_{2}-\sigma_{1}} \int_{\sigma_{1}}^{\sigma_{2}} \mathcal{P}\left(\mathfrak{d}_{1}\right) \mathcal{S}\left(\mathfrak{d}_{1}\right) d \mathfrak{d}_{1}-\frac{1}{\left(\sigma_{2}-\sigma_{1}\right)^{2}} \int_{\sigma_{1}}^{\sigma_{2}} \mathcal{P}\left(\mathfrak{d}_{1}\right) d \mathfrak{d}_{1} \int_{\sigma_{1}}^{\sigma_{2}} \mathcal{S}\left(\mathfrak{d}_{1}\right) d \mathfrak{d}_{1}\right| \\
& \quad \leq \frac{1}{4}(\mathcal{M}-m)(\mathcal{N}-n),
\end{aligned}
$$

where the constant $1 / 4$ cannot be improved.

Grüss inequality (1.1) connects the integral of the product of two functions with the product of their integrals. It is extensively identified that continuous and discrete cases of Grüss type variants play a considerable job in examining the qualitative conduct of differential and integral equations. Inspired by Grüss inequality (1.1), we intend to show modified versions of (1.1) by using generalized $\mathcal{K}$-fractional integrals. For the reason that such variants are supposed to be vital, the exploration has continued to develop the investigations for such kinds of variants. Our findings and their utilities appeared in a variety of academic papers (see [61-64]). Amongst such sorts of inequalities, the Grüss inequality is one of the most fascinating inequalities.

We are influenced to take a look at this inequality for the generalized $\mathcal{K}$-fractional integral. As a consequence, we obtain numerous fractional integral inequalities, such inequalities are worthwhile in the fields of fractional differential equations. Also we apply these fractional inequalities to find new versions for the generalized Riemann-Liouville fractional integral. In this sequel, we present some preliminary results in order to prove our main results later.

Definition 1.2 Let $\mathcal{P} \in L_{1}\left(\left[\sigma_{1}, \sigma_{2}\right]\right)$ (the Lebesgue space). Then the left- and right-sided generalized Riemann-Liouville fractional integrals of order $\rho>0$ are defined by

$$
\mathfrak{J}_{\sigma_{1}^{+}}^{\varrho} \mathcal{P}(\varrho)=\frac{1}{\Gamma(\rho)} \int_{\sigma_{1}}^{\varrho}(\varrho-\zeta)^{\rho-1} \mathcal{P}(\zeta) d \zeta, \quad \varrho>\sigma_{1}
$$

and

$$
\mathfrak{J}_{\sigma_{2}^{-}}^{\varrho} \mathcal{P}(\varrho)=\frac{1}{\Gamma(\rho)} \int_{\varrho}^{\sigma_{2}}(\zeta-\lambda)^{\rho-1} \mathcal{P}(\zeta) d \zeta, \quad \varrho<\sigma_{2}
$$

where $\Gamma(\cdot)$ is the gamma function $[65,66]$. 
Now, we give the definition of more general fractional integral, which is mainly due to Mubeen and Habibullah [18].

Definition 1.3 (see [18]) Let $\mathcal{P} \in L_{1}\left(\left[\sigma_{1}, \sigma_{2}\right]\right)$ (the Lebsegue space). Then the left-sided and right-sided $\mathcal{K}$-fractional integrals of order $\rho, \mathcal{K}>0$ are defined by

$$
\mathfrak{J}_{\sigma_{1}^{+}}^{\rho, \mathcal{K}} \mathcal{P}(\varrho)=\frac{1}{\mathcal{K} \Gamma_{\mathcal{K}}(\rho)} \int_{\sigma_{1}}^{\varrho}(\varrho-\zeta)^{\frac{\rho}{\mathcal{K}}-1} \mathcal{P}(\zeta) d \zeta \quad\left(\varrho>\sigma_{1}\right)
$$

and

$$
\mathfrak{J}_{\sigma_{2}}^{\rho, \mathcal{K}} \mathcal{P}(\varrho)=\frac{1}{\mathcal{K} \Gamma_{\mathcal{K}}(\rho)} \int_{\varrho}^{\sigma_{2}}(\zeta-\varrho)^{\rho}{ }^{-1} \mathcal{P}(\zeta) d \zeta \quad\left(\varrho<\sigma_{2}\right)
$$

Further, we demonstrate the concept of generalized Riemann-Liouville fractional integral as follows.

Definition 1.4 (see $[11,67])$ Let $\sigma_{1}, \sigma_{2} \in(-\infty,+\infty)$ such that $\sigma_{1}<\sigma_{2}$ and $\Psi(\zeta)$ be an increasing and positive monotone function on $\left(\sigma_{1}, \sigma_{2}\right]$. Then the left-sided and right-sided generalized Riemann-Liouville fractional integrals of a function $\mathcal{P}$ with respect to another function $\Psi$ of order $\rho>0$ are defined by

$$
\begin{aligned}
& \mathfrak{J}_{\sigma_{1}^{+}}^{\rho, \Psi} \mathcal{P}(\varrho)=\frac{1}{\Gamma(\varrho)} \int_{\sigma_{1}}^{\varrho} \Psi^{\prime}(\zeta)(\Psi(\varrho)-\Psi(\zeta))^{\rho-1} \mathcal{P}(\zeta) d \zeta \\
& \mathfrak{J}_{\sigma_{2}^{-}}^{\rho, \Psi} \mathcal{P}(\varrho)=\frac{1}{\Gamma(\rho)} \int_{\varrho}^{\sigma_{2}} \Psi^{\prime}(\zeta)(\Psi(\zeta)-\Psi(\varrho))^{\rho-1} \mathcal{P}(\zeta) d \zeta
\end{aligned}
$$

We define the more general form of generalized $\mathcal{K}$-fractional integral as follows.

Definition 1.5 Let $\sigma_{1}, \sigma_{2} \in(-\infty,+\infty)$ such that $\sigma_{1}<\sigma_{2}$ and $\Psi(\zeta)$ be an increasing and positive monotone function on $\left(\sigma_{1}, \sigma_{2}\right]$. Then the left-sided and right-sided generalized $\mathcal{K}$-fractional integrals of a function $\mathcal{P}$ with respect to another function $\Psi$ of order $\rho, \mathcal{K}>0$ are defined by

$$
\begin{aligned}
& \mathfrak{J}_{\Psi, \sigma_{1}^{+}}^{\rho, \mathcal{K}} \mathcal{P}(\varrho)=\frac{1}{\mathcal{K} \Gamma_{\mathcal{K}}(\rho)} \int_{\sigma_{1}}^{\varrho} \Psi^{\prime}(\zeta)(\Psi(\varrho)-\Psi(\zeta))^{\frac{\rho}{\mathcal{K}}-1} \mathcal{P}(\zeta) d \zeta \\
& \mathfrak{J}_{\Psi, \sigma_{2}^{-}}^{\rho, \mathcal{N}} \mathcal{P}(\varrho)=\frac{1}{\mathcal{K} \Gamma_{\mathcal{K}}(\rho)} \int_{\varrho}^{\sigma_{2}} \Psi^{\prime}(\zeta)(\Psi(\zeta)-\Psi(\varrho))^{\frac{\rho}{\mathcal{K}}-1} \mathcal{P}(\zeta) d \zeta
\end{aligned}
$$

where $\Gamma_{\mathcal{K}}$ is the $\mathcal{K}$-gamma function.

Remark 1.6 From Definition 1.5 we clearly see that Definition 1.2, Definition 1.3, and Definition 1.4 can be obtained if we take $\mathcal{K}=1, \Psi(\varrho)=\varrho$, and $\Psi(\varrho)=\varrho$ and $\mathcal{K}=1$, respectively.

Grüss type inequality and its useful consequences are investigated by Kacar et al. [68]. Motivated by [68], we provide new and novel results using generalized $\mathcal{K}$-fractional integral related to (1.1). Consequently, the effects furnished in this research paper are an extra generalization. 


\section{Main results}

Theorem 2.1 Let $\mathcal{K}, \rho, \delta>0, \mathcal{P}$ be a positive function on $[0, \infty)$ and $\Psi$ be an increasing and positive function on $[0, \infty)$ such that $\Psi^{\prime}(x)$ is continuous on $[0, \infty)$ with $\Psi(0)=0$. Suppose that there exist integrable functions $\Theta_{1}, \Theta_{2}$ on $[0, \infty)$ such that

$$
\Theta_{1}(\varrho) \leq \mathcal{P}(\varrho) \leq \Theta_{2}(\varrho)
$$

for all $\varrho \in[0, \infty)$. Then we have

$$
\begin{aligned}
& \mathfrak{J}_{\Psi, 0^{+}}^{\delta, \mathcal{K}} \Theta_{1}(\varrho) \mathfrak{J}_{\Psi, 0^{+}}^{\rho, \mathcal{K}} \mathcal{P}(\varrho)+\mathfrak{J}_{\Psi, 0^{+}}^{\rho, \mathcal{K}} \Theta_{2}(\varrho) \mathfrak{J}_{\Psi, 0^{+}}^{\delta, \mathcal{K}} \mathcal{P}(\varrho) \\
& \quad \geq \mathfrak{J}_{\Psi, 0^{+}}^{\rho, \mathcal{K}} \Theta_{2}(\varrho) \mathfrak{J}_{\Psi, 0^{+}}^{\delta, \mathcal{K}} \Theta_{1}(\varrho)+\mathfrak{J}_{\Psi, 0^{+}}^{\rho, \mathcal{K}} \mathcal{P}(\varrho) \mathfrak{J}_{\Psi, 0^{+}}^{\delta, \mathcal{K}} \mathcal{P}(\varrho)
\end{aligned}
$$

Proof Using (2.1), for all $\mathfrak{d}_{1} \geq 0, \mathfrak{d}_{2} \geq 0$, we have

$$
\begin{aligned}
& \left(\Theta_{2}\left(\mathfrak{d}_{1}\right)-\mathcal{P}\left(\mathfrak{d}_{1}\right)\right)\left(\mathcal{P}\left(\mathfrak{d}_{2}\right)-\Theta_{1}\left(\mathfrak{d}_{2}\right)\right) \geq 0 \\
& \Theta_{2}\left(\mathfrak{d}_{1}\right) \mathcal{P}\left(\mathfrak{d}_{2}\right)+\Theta_{1}\left(\mathfrak{d}_{2}\right) \mathcal{P}\left(\mathfrak{d}_{1}\right) \geq \Theta_{1}\left(\mathfrak{d}_{2}\right) \Theta_{2}\left(\mathfrak{d}_{1}\right)+\mathcal{P}\left(\mathfrak{d}_{1}\right) \mathcal{P}\left(\mathfrak{d}_{2}\right)
\end{aligned}
$$

If we multiply both sides of (2.3) by $\frac{\left(\Psi(\varrho)-\Psi\left(\mathfrak{d}_{1}\right)\right) \mathcal{K}^{-1} \Psi^{\prime}\left(\mathfrak{d}_{1}\right)}{\mathcal{K} \Gamma_{\mathcal{K}}(\rho)}$ and integrate with respect to $\mathfrak{d}_{1}$ on $(0, \varrho)$, we obtain

$$
\begin{aligned}
& \mathcal{P}\left(\mathfrak{d}_{2}\right) \frac{1}{\mathcal{K} \Gamma_{\mathcal{K}}(\rho)} \int_{0}^{\varrho}\left(\Psi(\varrho)-\Psi\left(\mathfrak{d}_{1}\right)\right)^{\frac{\rho}{\mathcal{K}}-1} \Psi^{\prime}\left(\mathfrak{d}_{1}\right) \Theta_{2}\left(\mathfrak{d}_{1}\right) d \mathfrak{d}_{1} \\
& \quad+\Theta_{1}\left(\mathfrak{d}_{2}\right) \frac{1}{\mathcal{K} \Gamma_{\mathcal{K}}(\rho)} \int_{0}^{\varrho}\left(\Psi(\varrho)-\Psi\left(\mathfrak{d}_{1}\right)\right)^{\frac{\rho}{\mathcal{K}}-1} \Psi^{\prime}\left(\mathfrak{d}_{1}\right) \mathcal{P}\left(\mathfrak{d}_{1}\right) d \mathfrak{d}_{1} \\
& \geq \Theta_{1}\left(\mathfrak{d}_{2}\right) \frac{1}{\mathcal{K} \Gamma_{\mathcal{K}}(\rho)} \int_{0}^{\varrho}\left(\Psi(\varrho)-\Psi\left(\mathfrak{d}_{1}\right)\right)^{\frac{\rho}{\mathcal{K}}-1} \Psi^{\prime}\left(\mathfrak{d}_{1}\right) \Theta_{2}\left(\mathfrak{d}_{1}\right) d \mathfrak{d}_{1} \\
& \quad+\mathcal{P}\left(\mathfrak{d}_{2}\right) \frac{1}{\mathcal{K} \Gamma_{\mathcal{K}}(\rho)} \int_{0}^{\varrho}\left(\Psi(\varrho)-\Psi\left(\mathfrak{d}_{1}\right)\right)^{\frac{\rho}{\mathcal{K}}-1} \Psi^{\prime}\left(\mathfrak{d}_{1}\right) \mathcal{P}\left(\mathfrak{d}_{1}\right) d \mathfrak{d}_{1},
\end{aligned}
$$

which can be written as

$$
\begin{aligned}
& \mathcal{P}\left(\mathfrak{d}_{2}\right) \mathfrak{J}_{\Psi, 0^{+}}^{\rho, \mathcal{K}} \Theta_{2}(\varrho)+\Theta_{1}\left(\mathfrak{d}_{2}\right) \mathfrak{J}_{\Psi, 0^{+}}^{\rho, \mathcal{K}} \mathcal{P}(\varrho) \\
& \left.\quad \geq \Theta_{1}\left(\mathfrak{d}_{2}\right) \mathfrak{J}_{\Psi, 0^{+}}^{\rho, \mathcal{K}} \Theta_{2}(\varrho)+\mathcal{P}\left(\mathfrak{d}_{2}\right)\right)_{\mathfrak{J}_{\Psi, 0^{+}}^{\rho}}^{\rho, \mathcal{K}} \mathcal{P}(\varrho) .
\end{aligned}
$$


$\mathfrak{d}_{2}$ on $(0, \varrho)$, we get

$$
\begin{aligned}
& \mathfrak{J}_{\Psi, 0^{+}}^{\delta, \mathcal{K}} \Theta_{1}(\varrho) \mathfrak{J}_{\Psi, 0^{+}}^{\rho, \mathcal{K}} \mathcal{P}(\varrho)+\mathfrak{J}_{\Psi, 0^{+}}^{\rho, \mathcal{K}} \Theta_{2}(\varrho) \mathfrak{J}_{\Psi, 0^{+}}^{\delta, \mathcal{K}} \mathcal{P}(\varrho) \\
& \quad \geq \mathfrak{J}_{\Psi, 0^{+}}^{\rho, \mathcal{K}} \Theta_{2}(\varrho) \mathfrak{J}_{\Psi, 0^{+}}^{\delta, \mathcal{K}} \Theta_{1}(\varrho)+\mathfrak{J}_{\Psi, 0^{+}}^{\rho, \mathcal{K}} \mathcal{P}(\varrho) \mathfrak{J}_{\Psi, 0^{+}}^{\delta, \mathcal{K}} \mathcal{P}(\varrho)
\end{aligned}
$$

This completes the proof of Theorem 2.1. 
Corollary 2.2 Let $\Psi(\varrho)=\varrho$. Then Theorem 2.1 leads to the inequality for $\mathcal{K}$-fractional integral as follows:

$$
\begin{aligned}
& \mathfrak{J}^{\delta, \mathcal{K}} \Theta_{1}(\varrho) \mathfrak{J}^{\rho, \mathcal{K}} \mathcal{P}(\varrho)+\mathfrak{J}^{\rho, \mathcal{K}} \Theta_{2}(\varrho) \mathfrak{J}^{\delta, \mathcal{K}} \mathcal{P}(\varrho) \\
& \quad \geq \mathfrak{J}^{\rho, \mathcal{K}} \Theta_{2}(\varrho) \mathfrak{J}^{\delta, \mathcal{K}} \Theta_{1}(\varrho)+\mathfrak{J}^{\rho, \mathcal{K}} \mathcal{P}(\varrho) \mathfrak{J}^{\delta, \mathcal{K}} \mathcal{P}(\varrho) .
\end{aligned}
$$

Corollary 2.3 Let $m, \mathcal{M} \in \mathcal{R}$ with $m<\mathcal{M} \in \mathcal{R}, \mathcal{K}, \rho, \delta>0$ and $\mathcal{P}$ be a positive function on $[0, \infty)$ such that $m \leq \mathcal{P}(\varrho) \leq \mathcal{M}$. Then one has

$$
\begin{aligned}
& m \frac{\Psi \frac{\delta}{\mathcal{K}}(\varrho)}{\Gamma_{\mathcal{K}}(\delta+\mathcal{K})} \mathfrak{J}_{\Psi, 0^{+}}^{\rho, \mathcal{K}} \mathcal{P}(\varrho)+\mathcal{M} \frac{\Psi \frac{\rho}{\mathcal{K}}(\varrho)}{\Gamma_{\mathcal{K}}(\rho+\mathcal{K})} \mathfrak{J}_{\Psi, 0^{+}}^{\delta, \mathcal{K}} \mathcal{P}(\varrho) \\
& \quad \geq m \mathcal{M} \frac{\Psi \frac{\delta}{\mathcal{K}}(\varrho)}{\Gamma_{\mathcal{K}}(\delta+\mathcal{K})} \frac{\Psi \frac{\rho}{\mathcal{K}}(\varrho)}{\Gamma_{\mathcal{K}}(\rho+\mathcal{K})}+\mathfrak{J}_{\Psi, 0^{+}}^{\rho, \mathcal{K}} \mathcal{P}(\varrho) \mathfrak{J}_{\Psi, 0^{+}}^{\delta, \mathcal{K}} \mathcal{P}(\varrho)
\end{aligned}
$$

Remark 2.4 Theorem 2.1, Corollary 2.2, and Corollary 2.3 lead to the following conclusions:

(1) If $\mathcal{K}=1$ in Theorem 2.1, then we get Theorem 2.11 in [68].

(2) If $\mathcal{K}=1$ and $\Psi(\varrho)=\varrho$ in Theorem 2.1, then we get Theorem 2 in [69].

(3) If $\mathcal{K}=1$ in Corollary 2.2, then we get Corollary 2.14 in [68].

(4) If $\mathcal{K}=1$ and $\Psi(\varrho)=\varrho$ in Corollary 2.3, then we obtain Corollary 3 in [69].

Theorem 2.5 Let $\mathcal{K}>0, \rho, \delta>0, \mathcal{P}$ and $\mathcal{S}$ be two positive functions on $[0, \infty)$, and $\Psi$ be an increasing and positive function on $[0, \infty)$ such that $\Psi(0)=0$ and $\Psi^{\prime}$ is continuous on $[0, \infty)$. Suppose that $(2.1)$ holds and there exist integrable functions $\varphi_{1}$ and $\varphi_{2}$ on $[0, \infty)$ such that

$$
\varphi_{1}(\varrho) \leq \mathcal{S}(\varrho) \leq \varphi_{2}(\varrho)
$$

for all $\varrho \in[0, \infty)$. Then we have four inequalities as follows:

$$
\begin{aligned}
& \text { (a) } \mathfrak{J}_{\Psi, 0^{+}}^{\delta, \mathcal{K}} \varphi_{1}(\varrho) \mathfrak{J}_{\Psi, 0^{+}}^{\rho, \mathcal{K}} \mathcal{P}(\varrho)+\mathfrak{J}_{\Psi, 0^{+}}^{\rho, \mathcal{K}} \Theta_{2}(\varrho) \mathfrak{J}_{\Psi, 0^{+}}^{\delta, \mathcal{K}} \mathcal{S}(\varrho) \\
& \left.\geq \mathfrak{J}_{\Psi, 0^{+}}^{\delta, \mathcal{K}} \varphi_{2}(\varrho) \mathfrak{J}_{\Psi, 0^{+}}^{\rho, \mathcal{K}} \Theta_{2}(\varrho)+\mathfrak{J}_{\Psi, 0^{+}}^{\rho} \mathcal{P}(\varrho)\right)_{\mathfrak{J}_{\Psi, 0^{+}}^{\delta, \mathcal{K}}} \mathcal{S}(\varrho), \\
& \text { (b) } \left.\mathfrak{J}_{\Psi, 0^{+}}^{\delta, \mathcal{K}} \Theta_{1}(\varrho) \tilde{J}_{\Psi, 0^{+}}^{\rho, \mathcal{K}} \mathcal{S}(\varrho)+\mathfrak{J}_{\Psi, 0^{+}}^{\rho, \mathcal{K}} \varphi_{2}(\varrho)\right)_{\mathfrak{J}_{\Psi, 0^{+}}^{\delta, \mathcal{K}}} \mathcal{P}(\varrho) \\
& \geq \mathfrak{J}_{\Psi, 0^{+}}^{\delta, \mathcal{K}} \Theta_{1}(\varrho) \mathfrak{J}_{\Psi, 0^{+}}^{\rho, \mathcal{K}} \varphi_{2}(\varrho)+\mathfrak{J}_{\Psi, 0^{+}}^{\delta, \mathcal{K}} \mathcal{P}(\varrho) \mathfrak{J}_{\Psi, 0^{+}}^{\rho, \mathcal{K}} \mathcal{S}(\varrho), \\
& \text { (c) } \mathfrak{J}_{\Psi, 0^{+}}^{\rho, \mathcal{K}} \Theta_{2}(\varrho) \mathfrak{J}_{\Psi, 0^{+}}^{\delta, \mathcal{K}} \varphi_{2}(\varrho)+\mathfrak{J}_{\Psi, 0^{+}}^{\rho, \mathcal{K}} \mathcal{P}(\varrho) \mathfrak{J}_{\Psi, 0^{+}}^{\delta, \mathcal{K}} \mathcal{S}(\varrho) \\
& \geq \mathfrak{J}_{\Psi, 0^{+}}^{\rho, \mathcal{K}} \Theta_{2}(\varrho) \mathfrak{J}_{\Psi, 0^{+}}^{\delta, \mathcal{K}} \mathcal{S}(\varrho)+\mathfrak{J}_{\Psi, 0^{+}}^{\delta, \mathcal{K}} \varphi_{2}(\varrho) \mathfrak{J}_{\Psi, 0^{+}}^{\rho, \mathcal{K}} \mathcal{P}(\varrho), \\
& \text { (d) } \mathfrak{J}_{\Psi, 0^{+}}^{\rho, \mathcal{K}} \Theta_{1}(\varrho) \mathfrak{J}_{\Psi, 0^{+}}^{\delta, \mathcal{K}} \varphi_{1}(\varrho)+\mathfrak{J}_{\Psi, 0^{+}}^{\rho, \mathcal{K}} \mathcal{P}(\varrho) \mathfrak{J}_{\Psi, 0^{+}}^{\delta, \mathcal{K}} \mathcal{S}(\varrho) \\
& \left.\geq \mathfrak{J}_{\Psi, 0^{+}}^{\rho, \mathcal{K}} \Theta_{1}(\varrho) \mathfrak{J}_{\Psi, 0^{+}}^{\delta, \mathcal{K}} \mathcal{S}(\varrho)+\mathfrak{J}_{\Psi, 0^{+}}^{\delta, \mathcal{K}} \varphi_{1}(\varrho)\right)_{\mathfrak{J}_{\Psi, 0^{+}}^{\rho, \mathcal{K}}} \mathcal{P}(\varrho) .
\end{aligned}
$$

Proof For any $\varrho \in[0, \infty)$, it follows from (2.1) and (2.6) that

$$
\left(\Theta_{2}\left(\mathfrak{d}_{1}\right)-\mathcal{P}\left(\mathfrak{d}_{1}\right)\right)\left(\mathcal{S}\left(\mathfrak{d}_{2}\right)-\varphi_{1}\left(\mathfrak{d}_{2}\right)\right) \geq 0
$$


and

$$
\Theta_{2}\left(\mathfrak{d}_{1}\right) \mathcal{S}\left(\mathfrak{d}_{2}\right)+\varphi_{1}\left(\mathfrak{d}_{2}\right) \mathcal{P}\left(\mathfrak{d}_{1}\right) \geq \varphi_{1}\left(\mathfrak{d}_{2}\right) \Theta_{2}\left(\mathfrak{d}_{1}\right)+\mathcal{P}\left(\mathfrak{d}_{1}\right) \mathcal{S}\left(\mathfrak{d}_{2}\right)
$$

Taking product on both sides of $(2.8)$ by $\frac{\left(\Psi(\varrho)-\Psi\left(\mathfrak{d}_{1}\right)\right) \frac{\rho}{\mathcal{K}}-1 \Psi^{\prime}\left(\mathfrak{d}_{1}\right)}{\mathcal{K} \Gamma_{\mathcal{K}}(\rho)}$ and integrating the resulting identity for the variable $\mathfrak{d}_{1}$ on $(0, \varrho)$ give

$$
\begin{aligned}
& \mathcal{S}\left(\mathfrak{d}_{2}\right) \frac{1}{K \Gamma_{k}(\rho)} \int_{0}^{\varrho}\left(\Psi(\varrho)-\Psi\left(\mathfrak{d}_{1}\right)\right)^{\frac{\rho}{\mathcal{K}}-1} \Psi^{\prime}\left(\mathfrak{d}_{1}\right) \Theta_{2}\left(\mathfrak{d}_{1}\right) d \mathfrak{d}_{1} \\
& \quad+\varphi_{1}\left(\mathfrak{d}_{2}\right) \frac{1}{\mathcal{K} \Gamma_{\mathcal{K}}(\rho)} \int_{0}^{\varrho}\left(\Psi(\varrho)-\Psi\left(\mathfrak{d}_{1}\right)\right)^{\frac{\rho}{\mathcal{K}}-1} \Psi^{\prime}\left(\mathfrak{d}_{1}\right) \mathcal{P}\left(\mathfrak{d}_{1}\right) d \mathfrak{d}_{1} \\
& \geq \varphi_{1}\left(\mathfrak{d}_{2}\right) \frac{1}{\mathcal{K} \Gamma_{\mathcal{K}}(\rho)} \int_{0}^{\varrho}\left(\Psi(\varrho)-\Psi\left(\mathfrak{d}_{1}\right)\right)^{\frac{\rho}{\mathcal{K}}-1} \Psi^{\prime}\left(\mathfrak{d}_{1}\right) \Theta_{2}\left(\mathfrak{d}_{1}\right) d \mathfrak{d}_{1} \\
& +\mathcal{S}\left(\mathfrak{d}_{2}\right) \frac{1}{\mathcal{K} \Gamma_{\mathcal{K}}(\rho)} \int_{0}^{\varrho}\left(\Psi(\varrho)-\Psi\left(\mathfrak{d}_{1}\right)\right)^{\frac{\rho}{\mathcal{K}}-1} \Psi^{\prime}\left(\mathfrak{d}_{1}\right) \mathcal{P}\left(\mathfrak{d}_{1}\right) d \mathfrak{d}_{1},
\end{aligned}
$$

which can be rewritten as

$$
\begin{aligned}
& \mathcal{S}\left(\mathfrak{d}_{2}\right) \mathfrak{J}_{\Psi, 0^{+}}^{\rho, \mathcal{K}} \Theta_{2}(\varrho)+\varphi_{1}\left(\mathfrak{d}_{2}\right) \mathfrak{J}_{\Psi, 0^{+}}^{\rho, \mathcal{K}} \mathcal{P}(\varrho) \\
& \quad \geq \varphi_{1}\left(\mathfrak{d}_{2}\right) \mathfrak{J}_{\Psi, 0^{+}}^{\rho, \mathcal{K}} \Theta_{2}(\varrho)+\mathcal{S}\left(\mathfrak{d}_{2}\right) \mathfrak{J}_{\Psi, 0^{+}}^{\rho, \mathcal{K}} \mathcal{P}(\varrho)
\end{aligned}
$$

Again, taking product on both sides of (2.9) by $\frac{\left.\left(\Psi(\varrho)-\Psi\left(\mathfrak{d}_{2}\right)\right)\right)^{\frac{\delta}{\mathcal{K}}}-1 \Psi^{\prime}\left(\mathfrak{d}_{2}\right)}{\mathcal{K} \Gamma_{\mathcal{K}}(\delta)}$ and integrating the resulting identity for the variable $\mathfrak{d}_{2}$ on $(0, \varrho)$, we obtain

$$
\begin{aligned}
& \mathfrak{J}_{\Psi, 0^{+}}^{\delta, \mathcal{K}} \varphi_{1}(\varrho) \mathfrak{J}_{\Psi, 0^{+}}^{\rho, \mathcal{K}} \mathcal{P}(\varrho)+\mathfrak{J}_{\Psi, 0^{+}}^{\rho, \mathcal{K}} \Theta_{2}(\varrho) \mathfrak{J}_{\Psi, 0^{+}}^{\delta, \mathcal{K}} \mathcal{S}(\varrho) \\
& \quad \geq \mathfrak{J}_{\Psi, 0^{+}}^{\delta, \mathcal{K}} \varphi_{1}(\varrho) \mathfrak{J}_{\Psi, 0^{+}}^{\rho, \mathcal{K}} \Theta_{2}(\varrho)+\mathfrak{J}_{\Psi, 0^{+}}^{\rho, \mathcal{K}} \mathcal{P}(\varrho) \mathfrak{J}_{\Psi, 0^{+}}^{\delta, \mathcal{K}} \mathcal{S}(\varrho)
\end{aligned}
$$

This proves part (a). To prove parts (b)-(d), we consider the subsequent inequalities:

(b) $\left(\varphi_{2}\left(\mathfrak{d}_{1}\right)-\mathcal{S}\left(\mathfrak{d}_{1}\right)\right)\left(\mathcal{P}\left(\mathfrak{d}_{2}\right)-\Theta_{1}\left(\mathfrak{d}_{2}\right)\right) \geq 0$,

(c) $\left(\Theta_{2}\left(\mathfrak{d}_{1}\right)-\mathcal{P}\left(\mathfrak{d}_{1}\right)\right)\left(\mathcal{S}\left(\mathfrak{d}_{2}\right)-\varphi_{2}\left(\mathfrak{d}_{2}\right)\right) \leq 0$,

(d) $\quad\left(\Theta_{1}\left(\mathfrak{d}_{1}\right)-\mathcal{P}\left(\mathfrak{d}_{1}\right)\right)\left(\mathcal{S}\left(\mathfrak{d}_{2}\right)-\varphi_{1}\left(\mathfrak{d}_{2}\right)\right) \leq 0$.

We use similar arguments as those in the proof of part (a) to get the rest of the inequalities.

The following inequalities are special cases of Theorem 2.5.

Corollary 2.6 Let $\mathcal{K}>0, \rho, \delta>0$, and $\mathcal{P}$ and $\mathcal{S}$ be two positive functions on $[0, \infty)$. Suppose that there exist real constants $m, \mathcal{M}, n, \mathcal{N}$ such that

$$
m \leq \mathcal{P}(\varrho) \leq \mathcal{M}, \quad n \leq \mathcal{S}(\varrho) \leq \mathcal{N}
$$

for all $\varrho \in[0, \infty)$. Then we obtain

(i) $n \frac{\Psi^{\frac{\delta}{\mathcal{K}}}(\varrho)}{\Gamma_{\mathcal{K}}(\delta+\mathcal{K})} \mathfrak{J}_{\Psi, 0^{+}}^{\rho, \mathcal{K}} \mathcal{P}(\varrho)+\mathcal{M} \frac{\Psi \frac{\rho}{\mathcal{K}}(\varrho)}{\Gamma_{\mathcal{K}}(\rho+\mathcal{K})} \mathfrak{J}_{\Psi, 0^{+}}^{\delta, \mathcal{K}} \mathcal{S}(\varrho)$ 


$$
\geq n \mathcal{M} \frac{\Psi^{\frac{\delta}{\mathcal{K}}}(\varrho)}{\Gamma_{\mathcal{K}}(\delta+\mathcal{K})} \frac{\Psi^{\stackrel{\rho}{\mathcal{K}}}(\varrho)}{\Gamma_{\mathcal{K}}(\rho+\mathcal{K})}+\mathfrak{J}_{\Psi, 0^{+}}^{\rho, \mathcal{K}} \mathcal{P}(\varrho) \mathfrak{J}_{\Psi, 0^{+}}^{\delta, \mathcal{K}} \mathcal{S}(\varrho)
$$

(ii) $\quad m \frac{\Psi \stackrel{\delta}{\mathcal{K}}(\varrho)}{\Gamma_{\mathcal{K}}(\delta+\mathcal{K})} \mathfrak{J}_{\Psi, 0^{+}}^{\rho, \mathcal{K}} \mathcal{S}(\varrho)+\mathcal{N} \frac{\Psi \frac{\rho}{\mathcal{K}}(\varrho)}{\Gamma_{\mathcal{K}}(\rho+\mathcal{K})} \mathfrak{J}_{\Psi, 0^{+}}^{\delta, \mathcal{K}} \mathcal{P}(\varrho)$

$$
\geq m \mathcal{N} \frac{\Psi \frac{\delta}{\mathcal{K}}(\varrho)}{\Gamma_{\mathcal{K}}(\delta+\mathcal{K})} \frac{\Psi \frac{\rho}{\mathcal{K}}(\varrho)}{\Gamma_{\mathcal{K}}(\rho+\mathcal{K})}+\mathfrak{J}_{\Psi, 0^{+}}^{\delta, \mathcal{K}} \mathcal{P}(\varrho) \mathfrak{J}_{\Psi, 0^{+}}^{\rho, \mathcal{K}} \mathcal{S}(\varrho)
$$

(iii) $\quad \mathcal{N} \mathcal{M} \frac{\Psi \frac{\delta}{\mathcal{K}}(\varrho)}{\Gamma_{\mathcal{K}}(\delta+\mathcal{K})} \frac{\Psi \frac{\rho}{\mathcal{K}}(\varrho)}{\Gamma_{\mathcal{K}}(\rho+\mathcal{K})}+\mathfrak{J}_{\Psi, 0^{+}}^{\rho, \mathcal{K}} \mathcal{P}(\varrho) \mathfrak{J}_{\Psi, 0^{+}}^{\delta, \mathcal{K}} \mathcal{S}(\varrho)$

$$
\geq \mathcal{M} \frac{\Psi \frac{\rho}{\mathcal{K}}(\varrho)}{\Gamma\left(\Gamma_{\mathcal{K}}(\rho+\mathcal{K})\right.} \mathfrak{J}_{\Psi, 0^{+}}^{\delta, \mathcal{K}} \mathcal{S}(\varrho)+\mathcal{N} \frac{\Psi \frac{\delta}{\mathcal{K}}(\varrho)}{\Gamma_{\mathcal{K}}(\delta+\mathcal{K})} \mathfrak{J}_{\Psi, 0^{+}}^{\rho, \mathcal{K}} \mathcal{P}(\varrho)
$$

(iv) $n m \frac{\Psi \frac{\delta}{\mathcal{K}}(\varrho)}{\Gamma_{\mathcal{K}}(\delta+\mathcal{K})} \frac{\Psi \frac{\rho}{\mathcal{K}}(\varrho)}{\Gamma_{\mathcal{K}}(\rho+\mathcal{K})}+\mathfrak{J}_{\Psi, 0^{+}}^{\rho, \mathcal{K}} \mathcal{P}(\varrho) \mathfrak{J}_{\Psi, 0^{+}}^{\delta, \mathcal{K}} \mathcal{S}(\varrho)$

$$
\geq m \frac{\Psi \stackrel{\rho}{\mathcal{K}}(\varrho)}{\Gamma_{\mathcal{K}}(\rho+\mathcal{K})} \mathfrak{J}_{\Psi, 0^{+}}^{\delta, \mathcal{K}} \mathcal{S}(\varrho)+n \frac{\Psi^{\frac{\delta}{\mathcal{K}}}(\varrho)}{\Gamma_{\mathcal{K}}(\delta+\mathcal{K})} \mathfrak{J}_{\Psi, 0^{+}}^{\rho, \mathcal{K}} \mathcal{P}(\varrho) .
$$

Corollary 2.7 Let $\mathcal{K}>0, \rho, \delta>0, \mathcal{P}, \mathcal{S} \in L_{1}[0, \infty]$ and $\Psi(\varrho)=\varrho$. Suppose that there exist real constants $m, \mathcal{M}, n, \mathcal{N}$ such that

$$
m \leq \mathcal{P}(\varrho) \leq \mathcal{M}, \quad n \leq \mathcal{S}(\varrho) \leq \mathcal{N}
$$

for all $\varrho \in[0, \infty)$, then one has

(a) $\mathfrak{J}^{\delta, \mathcal{K}} \varphi_{1}(\varrho) \mathfrak{J}^{\rho, \mathcal{K}} \mathcal{P}(\varrho)+\mathfrak{J}^{\rho, \mathcal{K}} \Theta_{2}(\varrho) \mathfrak{J}^{\delta, \mathcal{K}} \mathcal{S}(\varrho)$

$$
\geq \mathfrak{J}^{\delta, \mathcal{K}} \varphi_{1}(\varrho) \mathfrak{J}^{\rho, \mathcal{K}} \Theta_{2}(\varrho)+\mathfrak{J}^{\rho, \mathcal{K}} \mathcal{P}(\varrho) \mathfrak{J}^{\delta, \mathcal{K}} \mathcal{S}(\varrho),
$$

(b) $\mathfrak{J}^{\delta, \mathcal{K}} \Theta_{1}(\varrho) \mathfrak{J}^{\rho, \mathcal{K}} \mathcal{S}(\varrho)+\mathfrak{J}^{\rho, \mathcal{K}} \varphi_{2}(\varrho) \mathfrak{J}^{\delta, \mathcal{K}} \mathcal{P}(\varrho)$

$$
\geq \mathfrak{J}^{\delta, \mathcal{K}} \Theta_{1}(\varrho) \mathfrak{J}^{\rho, \mathcal{K}} \varphi_{2}(\varrho)+\mathfrak{J}^{\delta, \mathcal{K}} \mathcal{P}(\varrho) \mathfrak{J}^{\rho, \mathcal{K}} \mathcal{S}(\varrho),
$$

(c) $\mathfrak{J}^{\rho, \mathcal{K}} \Theta_{2}(\varrho) \mathfrak{J}^{\delta, \mathcal{K}} \varphi_{2}(\varrho)+\mathfrak{J}^{\rho, \mathcal{K}} \mathcal{P}(\varrho) \mathfrak{J}^{\delta, \mathcal{K}} \mathcal{S}(\varrho)$

$$
\geq \mathfrak{J}^{\rho, \mathcal{K}} \Theta_{2}(\varrho) \mathfrak{J}^{\delta, \mathcal{K}} \mathcal{S}(\varrho)+\mathfrak{J}^{\delta, \mathcal{K}} \varphi_{2}(\varrho) \mathfrak{J}^{\rho, \mathcal{K}} \mathcal{P}(\varrho),
$$

(d) $\mathfrak{J}^{\rho, \mathcal{K}} \Theta_{1}(\varrho) \mathfrak{J}^{\delta, \mathcal{K}} \varphi_{1}(\varrho)+\mathfrak{J}^{\rho, \mathcal{K}} \mathcal{P}(\varrho) \mathfrak{J}^{\delta, \mathcal{K}} \mathcal{S}(\varrho)$

$$
\geq \mathfrak{J}^{\rho, \mathcal{K}} \Theta_{1}(\varrho) \mathfrak{J}^{\delta, \mathcal{K}} \mathcal{S}(\varrho)+\mathfrak{J}^{\delta, \mathcal{K}} \varphi_{1}(\varrho) \mathfrak{J}^{\rho, \mathcal{K}} \mathcal{P}(\varrho)
$$

Remark 2.8 From Theorem 2.5, and Corollaries 2.6 and 2.7 we get four conclusions as follows:

(1) If $\mathcal{K}=1$, then Theorem 2.5 leads to Theorem 2.15 in [68].

(2) If $\mathcal{K}=1$ and $\Psi(\varrho)=\varrho$, then Theorem 2.5 gives Theorem 5 in [69].

(3) If $\mathcal{K}=1$, then Corollary 2.6 becomes Corollary 2.16 in [68].

(4) If $\mathcal{K}=1$ and $\Psi(\varrho)=\varrho$, then Corollary 6 in [69] can be derived from Corollary 2.7.

Lemma 2.9 Let $\mathcal{K}>0, \rho, \delta>0, \mathcal{P}$ be a positive function on $[0, \infty), \Theta_{1}, \Theta_{2}$ be two integrable functions on $[0, \infty)$, and $\Psi$ be an increasing and positive function on $[0, \infty)$ such 
that $\Psi(0)=0$ and $\Psi^{\prime}$ is continuous on $[0, \infty)$. If $(2.1)$ holds, then

$$
\begin{aligned}
& \frac{\Psi \stackrel{\rho}{\mathcal{K}}(\varrho)}{\Gamma_{\mathcal{K}}(\rho+\mathcal{K})} \mathfrak{J}_{\Psi, 0^{+}}^{\rho, \mathcal{K}} \mathcal{P}^{2}(\varrho)-\left(\mathfrak{J}_{\Psi, 0^{+}}^{\rho, \mathcal{K}} \mathcal{P}(\varrho)\right)^{2} \\
& =\left(\mathfrak{J}_{\Psi, 0^{+}}^{\rho, \mathcal{K}} \Theta_{2}(\varrho)-\mathfrak{J}_{\Psi, 0^{+}}^{\rho, \mathcal{K}} \mathcal{P}(\varrho)\right)\left(\mathfrak{J}_{\Psi, 0^{+}}^{\rho, \mathcal{K}} \mathcal{P}(\varrho)-\mathfrak{J}_{\Psi, 0^{+}}^{\rho, \mathcal{K}} \Theta_{1}(\varrho)\right) \\
& -\frac{\Psi \stackrel{\rho}{\mathcal{K}}(\varrho)}{\Gamma_{\mathcal{K}}(\rho+\mathcal{K})}\left(\mathfrak{J}_{\Psi, 0^{+}}^{\rho, \mathcal{K}} \Theta_{2}(\varrho)-\mathfrak{J}_{\Psi, 0^{+}}^{\rho, \mathcal{K}} \mathcal{P}(\varrho)\right)\left(\mathfrak{J}_{\Psi, 0^{+}}^{\rho, \mathcal{K}} \mathcal{P}(\varrho)-\mathfrak{J}_{\Psi, 0^{+}}^{\rho, \mathcal{K}} \Theta_{1}(\varrho)\right) \\
& +\frac{\Psi \stackrel{\rho}{\mathcal{K}}(\varrho)}{\Gamma_{\mathcal{K}}(\rho+\mathcal{K})} \mathfrak{J}_{\Psi, 0^{+}}^{\rho, \mathcal{K}}\left(\Theta_{1}(\varrho) \mathcal{P}(\varrho)\right)-\mathfrak{J}_{\Psi, 0^{+}}^{\rho, \mathcal{K}} \Theta_{1}(\varrho) \mathfrak{J}_{\Psi, 0^{+}}^{\rho, \mathcal{K}} \mathcal{P}(\varrho) \\
& +\frac{\Psi \stackrel{\rho}{\mathcal{K}}(\varrho)}{\Gamma_{\mathcal{K}}(\rho+\mathcal{K})} \mathfrak{J}_{\Psi, 0^{+}}^{\rho, \mathcal{K}}\left(\Theta_{2}(\varrho) \mathcal{P}(\varrho)\right)-\mathfrak{J}_{\Psi, 0^{+}}^{\rho, \mathcal{K}} \Theta_{2}(\varrho) \mathfrak{J}_{\Psi, 0^{+}}^{\rho, \mathcal{K}} \mathcal{P}(\varrho) \\
& +\mathfrak{J}_{\Psi, 0^{+}}^{\rho, \mathcal{K}}\left(\Theta_{1}(\varrho)\right) \mathfrak{J}_{\Psi, 0^{+}}^{\rho, \mathcal{K}}\left(\Theta_{2}(\varrho)\right)-\frac{\Psi \stackrel{\rho}{\mathcal{K}}(\varrho)}{\Gamma_{\mathcal{K}}(\rho+\mathcal{K})} \mathfrak{J}_{\Psi, 0^{+}}^{\rho, \mathcal{K}}\left(\Theta_{1}(\varrho) \Theta_{2}(\varrho)\right) .
\end{aligned}
$$

Proof Since $\mathfrak{d}_{1}, \mathfrak{d}_{2}>0$, we have

$$
\begin{aligned}
\left(\Theta_{2}\left(\mathfrak{d}_{2}\right)-\mathcal{P}\left(\mathfrak{d}_{2}\right)\right)\left(\mathcal{P}\left(\mathfrak{d}_{1}\right)-\Theta_{1}\left(\mathfrak{d}_{1}\right)\right)+\left(\Theta_{2}\left(\mathfrak{d}_{1}\right)-\mathcal{P}\left(\mathfrak{d}_{1}\right)\right)\left(\mathcal{P}\left(\mathfrak{d}_{2}\right)-\Theta_{1}\left(\mathfrak{d}_{2}\right)\right) \\
\quad-\left(\Theta_{2}\left(\mathfrak{d}_{1}\right)-\mathcal{P}\left(\mathfrak{d}_{1}\right)\right)\left(\mathcal{P}\left(\mathfrak{d}_{1}\right)-\Theta_{1}\left(\mathfrak{d}_{1}\right)\right)-\left(\Theta_{2}\left(\mathfrak{d}_{2}\right)-\mathcal{P}\left(\mathfrak{d}_{2}\right)\right)\left(\mathcal{P}\left(\mathfrak{d}_{2}\right)-\Theta_{1}\left(\mathfrak{d}_{2}\right)\right) \\
=\mathcal{P}^{2}\left(\mathfrak{d}_{1}\right)+\mathcal{P}^{2}\left(\mathfrak{d}_{2}\right)-2 \mathcal{P}\left(\mathfrak{d}_{1}\right) \mathcal{P}\left(\mathfrak{d}_{2}\right)+\Theta_{2}\left(\mathfrak{d}_{2}\right) \mathcal{P}\left(\mathfrak{d}_{1}\right)+\Theta_{1}\left(\mathfrak{d}_{1}\right) \mathcal{P}\left(\mathfrak{d}_{2}\right) \\
\quad-\Theta_{1}\left(\mathfrak{d}_{1}\right) \Theta_{2}\left(\mathfrak{d}_{2}\right)+\Theta_{2}\left(\mathfrak{d}_{1}\right) \mathcal{P}\left(\mathfrak{d}_{2}\right)+\Theta_{1}\left(\mathfrak{d}_{2}\right) \mathcal{P}\left(\mathfrak{d}_{1}\right)-\Theta_{1}\left(\mathfrak{d}_{2}\right) \Theta_{2}\left(\mathfrak{d}_{1}\right) \\
\quad-\Theta_{2}\left(\mathfrak{d}_{1}\right) \mathcal{P}\left(\mathfrak{d}_{1}\right)+\Theta_{1}\left(\mathfrak{d}_{1}\right) \Theta_{2}\left(\mathfrak{d}_{1}\right)-\Theta_{1}\left(\mathfrak{d}_{1}\right) \mathcal{P}\left(\mathfrak{d}_{1}\right)-\Theta_{2}\left(\mathfrak{d}_{2}\right) \mathcal{P}\left(\mathfrak{d}_{2}\right) \\
\quad+\Theta_{1}\left(\mathfrak{d}_{2}\right) \Theta_{2}\left(\mathfrak{d}_{2}\right)-\Theta_{1}\left(\mathfrak{d}_{2}\right) \mathcal{P}\left(\mathfrak{d}_{2}\right) .
\end{aligned}
$$

Taking product on both sides of $(2.12)$ by $\frac{1}{\mathcal{K} \Gamma_{\mathcal{K}}(\rho)}\left(\Psi(\varrho)-\Psi\left(\mathfrak{d}_{1}\right)\right)^{\frac{\rho}{\mathcal{K}}-1} \Psi^{\prime}\left(\mathfrak{d}_{1}\right)$ and integrating the resulting identity for the variable $\mathfrak{d}_{1}$ on $(0, \varrho)$, we get

$$
\begin{aligned}
& \left(\Theta_{2}\left(\mathfrak{d}_{2}\right)-\mathcal{P}\left(\mathfrak{d}_{2}\right)\right)\left(\mathfrak{J}_{\Psi, 0^{+}}^{\rho, \mathcal{K}} \mathcal{P}(\varrho)-\mathfrak{J}_{\Psi, 0^{+}}^{\rho, \mathcal{K}} \Theta_{1}(\varrho)\right) \\
& +\left(\mathfrak{J}_{\Psi, 0^{+}}^{\rho, \mathcal{K}} \Theta_{2}(\varrho)-\mathfrak{J}_{\Psi, 0^{+}}^{\rho, \mathcal{K}} \mathcal{P}(\varrho)\right)\left(\mathcal{P}\left(\mathfrak{d}_{2}\right)-\Theta_{1}\left(\mathfrak{d}_{2}\right)\right) \\
& -\mathfrak{J}_{\Psi, 0^{+}}^{\rho, \mathcal{K}}\left(\left(\Theta_{2}(\varrho)-\mathcal{P}(\varrho)\right)\left(\mathcal{P}(\varrho)-\Theta_{1}(\varrho)\right)\right) \\
& -\left(\Theta_{2}\left(\mathfrak{d}_{2}\right)-\mathcal{P}\left(\mathfrak{d}_{2}\right)\right)\left(\mathcal{P}\left(\mathfrak{d}_{2}\right)-\Theta_{1}\left(\mathfrak{d}_{2}\right)\right) \frac{\Psi^{\stackrel{\rho}{\mathcal{K}}}(\varrho)}{\Gamma_{\mathcal{K}}(\rho+\mathcal{K})} \\
& =\mathfrak{J}_{\Psi, 0^{+}}^{\rho, \mathcal{K}} \mathcal{P}^{2}(\varrho)+\frac{\Psi \stackrel{\rho}{\mathcal{K}}(\varrho)}{\Gamma_{\mathcal{K}}(\varrho+\mathcal{K})} \mathcal{P}^{2}\left(\mathfrak{d}_{2}\right) \\
& -2 \mathcal{P}\left(\mathfrak{d}_{2}\right) \mathfrak{J}_{\Psi, 0^{+}}^{\rho, \mathcal{K}} \mathcal{P}(\varrho)+\Theta_{2}\left(\mathfrak{d}_{2}\right) \mathfrak{J}_{\Psi, 0^{+}}^{\rho, \mathcal{K}} \mathcal{P}(\varrho)+\mathcal{P}\left(\mathfrak{d}_{2}\right) \mathfrak{J}_{\Psi, 0^{+}}^{\rho, \mathcal{K}} \Theta_{1}(\varrho) \\
& -\Theta_{2}\left(\mathfrak{d}_{2}\right) \mathfrak{J}_{\Psi, 0^{+}}^{\rho, \mathcal{K}} \Theta_{1}(\varrho)+\mathcal{P}\left(\mathfrak{d}_{2}\right) \mathfrak{J}_{\Psi, 0^{+}}^{\rho, \mathcal{K}} \Theta_{2}(\varrho) \\
& +\Theta_{1}\left(\mathfrak{d}_{2}\right) \mathfrak{J}_{\Psi, 0^{+}}^{\rho, \mathcal{K}} \mathcal{P}(\varrho)-\Theta_{1}\left(\mathfrak{d}_{2}\right) \mathfrak{J}_{\Psi, 0^{+}}^{\rho, \mathcal{K}} \Theta_{2}(\varrho) \\
& -\mathfrak{J}_{\Psi, 0^{+}}^{\rho, \mathcal{K}}\left(\Theta_{2}(\varrho) \mathcal{P}(\varrho)\right)+\mathfrak{J}_{\Psi, 0^{+}}^{\rho, \mathcal{K}}\left(\Theta_{1}(\varrho) \Theta_{2}(\varrho)\right)-\mathfrak{J}_{\Psi, 0^{+}}^{\rho, \mathcal{K}}\left(\Theta_{1}(\varrho) \mathcal{P}(\varrho)\right) \\
& -\Theta_{2}\left(\mathfrak{d}_{2}\right) \mathcal{P}\left(\mathfrak{d}_{2}\right) \frac{\Psi^{\stackrel{\rho}{\mathcal{K}}}(\varrho)}{\Gamma_{\mathcal{K}}(\rho+\mathcal{K})}+\Theta_{1}\left(\mathfrak{d}_{2}\right) \Theta_{2}\left(\mathfrak{d}_{2}\right) \frac{\Psi^{\stackrel{\rho}{\mathcal{K}}}(\varrho)}{\Gamma_{\mathcal{K}}(\rho+\mathcal{K})} \\
& -\Theta_{1}\left(\mathfrak{d}_{2}\right) \mathcal{P}\left(\mathfrak{d}_{2}\right) \frac{\Psi^{\stackrel{\rho}{\mathcal{K}}}(\varrho)}{\Gamma_{\mathcal{K}}(\rho+\mathcal{K})} \text {. }
\end{aligned}
$$


Taking product on both sides of (2.13) by $\frac{1}{\mathcal{K} \Gamma_{\mathcal{K}}(\rho)}\left(\Psi(\varrho)-\Psi\left(\mathfrak{d}_{2}\right)\right)^{\frac{\rho}{\mathcal{K}}-1} \Psi^{\prime}\left(\mathfrak{d}_{2}\right)$ and integrating the resulting identity for the variable $\mathfrak{d}_{2}$ on $(0, \varrho)$, we get

$$
\begin{aligned}
& \left(\mathfrak{J}_{\Psi, 0^{+}}^{\rho, \mathcal{K}} \Theta_{2}(\varrho)-\mathfrak{J}_{\Psi, 0^{+}}^{\rho, \mathcal{K}} \mathcal{P}(\varrho)\right)\left(\mathfrak{J}_{\Psi, 0^{+}}^{\rho, \mathcal{K}} \mathcal{P}(\varrho)-\mathfrak{J}_{\Psi, 0^{+}}^{\rho, \mathcal{K}} \Theta_{1}(\varrho)\right) \\
& +\left(\mathfrak{J}_{\Psi, 0^{+}}^{\rho, \mathcal{K}} \Theta_{2}(\varrho)-\mathfrak{J}_{\Psi, 0^{+}}^{\rho, \mathcal{K}} \mathcal{P}(\varrho)\right)\left(\mathfrak{J}_{\Psi, 0^{+}}^{\rho, \mathcal{K}} \mathcal{P}(\varrho)-\mathfrak{J}_{\Psi, 0^{+}}^{\rho, \mathcal{K}} \Theta_{1}(\varrho)\right) \\
& -\mathfrak{J}_{\Psi, 0^{+}}^{\rho, \mathcal{K}}\left(\Theta_{2}(\varrho)-\mathcal{P}(\varrho)\right)\left(\mathcal{P}(\varrho)-\Theta_{1}(\varrho)\right) \frac{\Psi \frac{\rho}{\mathcal{K}}}{\Gamma_{\mathcal{K}}(\varrho+\mathcal{K})} \\
& -\left(\mathfrak{J}_{\Psi, 0^{+}}^{\rho, \mathcal{K}(\varrho)} \Theta_{2}(\varrho)-\mathfrak{J}_{\Psi, 0^{+}}^{\rho, \mathcal{K}} \mathcal{P}(\varrho)\right)\left(\mathfrak{J}_{\Psi, 0^{+}}^{\rho, \mathcal{K}} \mathcal{P}(\varrho)-\mathfrak{J}_{\Psi, 0^{+}}^{\rho, \mathcal{K}} \Theta_{1}(\varrho)\right) \frac{\Psi \stackrel{\rho}{\mathcal{K}}(\varrho)}{\Gamma_{\mathcal{K}}(\rho+\mathcal{K})} \\
& =\mathfrak{J}_{\Psi, 0^{+}}^{\rho, \mathcal{K}} \mathcal{P}^{2}(\varrho) \frac{\Psi \stackrel{\rho}{\mathcal{K}}(\varrho)}{\Gamma_{\mathcal{K}}(\rho+\mathcal{K})}+\frac{\Psi \stackrel{\rho}{\mathcal{K}}(\varrho)}{\Gamma_{\mathcal{K}}(\rho+\mathcal{K})} \mathfrak{J}_{\Psi, 0^{+}}^{\rho, \mathcal{K}} \mathcal{P}^{2}\left(\mathfrak{d}_{2}\right)-2 \mathfrak{J}_{\Psi, 0^{+}}^{\rho, \mathcal{K}} \mathcal{P}(\varrho) \mathfrak{J}_{\Psi, 0^{+}}^{\rho, \mathcal{K}} \mathcal{P}(\varrho) \\
& +\mathfrak{J}_{\Psi, 0^{+}}^{\rho, \mathcal{K}} \Theta_{2}(\varrho) \mathfrak{J}_{\Psi, 0^{+}}^{\rho, \mathcal{K}} \mathcal{P}(\varrho)+\mathfrak{J}_{\Psi, 0^{+}}^{\rho, \mathcal{K}} \mathcal{P}(\varrho) \mathfrak{J}_{\Psi, 0^{+}}^{\rho, \mathcal{K}} \Theta_{1}(\varrho)-\mathfrak{J}_{\Psi, 0^{+}}^{\rho, \mathcal{K}} \Theta_{2}(\varrho) \mathfrak{J}_{\Psi, 0^{+}}^{\rho, \mathcal{K}} \Theta_{1}(\varrho) \\
& +\mathfrak{J}_{\Psi, 0^{+}}^{\rho, \mathcal{K}} \mathcal{P}(\varrho) \mathfrak{J}_{\Psi, 0^{+}}^{\rho, \mathcal{K}} \Theta_{2}(\varrho)+\mathfrak{J}_{\Psi, 0^{+}}^{\rho, \mathcal{K}} \Theta_{1}(\varrho) \mathfrak{J}_{\Psi, 0^{+}}^{\rho, \mathcal{K}} \mathcal{P}(\varrho)-\mathfrak{J}_{\Psi, 0^{+}}^{\rho, \mathcal{K}} \Theta_{1}(\varrho) \mathfrak{J}_{\Psi, 0^{+}}^{\rho, \mathcal{K}} \Theta_{2}(\varrho) \\
& -\frac{\Psi \frac{\rho}{\mathcal{K}}(\varrho)}{\Gamma_{\mathcal{K}}(\rho+\mathcal{K})} \mathfrak{J}_{\Psi, 0^{+}}^{\rho, \mathcal{K}}\left(\Theta_{2}(\varrho) \mathcal{P}(\varrho)\right)+\frac{\Psi \frac{\rho}{\mathcal{K}}(\varrho)}{\Gamma_{\mathcal{K}}(\rho+\mathcal{K})} \mathfrak{J}_{\Psi, 0^{+}}^{\rho, \mathcal{K}}\left(\Theta_{1}(\varrho) \Theta_{2}(\varrho)\right) \\
& -\frac{\Psi \frac{\rho}{\mathcal{K}}(\varrho)}{\Gamma_{\mathcal{K}}(\rho+\mathcal{K})} \mathfrak{J}_{\Psi}^{\rho, \mathcal{K}}\left(\Theta_{1}(\varrho) \mathcal{P}(\varrho)\right)-\frac{\Psi \frac{\rho}{\mathcal{K}}(\varrho)}{\Gamma_{\mathcal{K}}(\rho+\mathcal{K})} \mathfrak{J}_{\Psi, 0^{+}}^{\rho, \mathcal{K}}\left(\Theta_{2}(\varrho) \mathcal{P}(\varrho)\right) \\
& +\frac{\Psi^{\rho} \mathcal{K}(\varrho)}{\Gamma_{\mathcal{K}}(\rho+\mathcal{K})} \mathfrak{J}_{\Psi, 0^{+}}^{\rho, \mathcal{K}}\left(\Theta_{1}(\varrho) \Theta_{2}(\varrho)\right)-\frac{\Psi \stackrel{\rho}{\mathcal{K}}(\varrho)}{\Gamma_{\mathcal{K}}(\rho+\mathcal{K})} \mathfrak{J}_{\Psi, 0^{+}}^{\rho, \mathcal{K}}\left(\Theta_{1}(\varrho) \mathcal{P}(\varrho)\right) .
\end{aligned}
$$

This is the proof of Lemma 2.9 .

Corollary 2.10 Let $\Psi(\varrho)=\varrho$ in Lemma 2.9 , then we have the $\mathcal{K}$-fractional integral inequality

$$
\begin{aligned}
& \frac{\varrho^{\frac{\rho}{\mathcal{K}}}}{\Gamma_{\mathcal{K}}(\rho+\mathcal{K})} \mathfrak{J}^{\rho, \mathcal{K}} \mathcal{P}^{2}(\varrho)-\left(\mathfrak{J}^{\rho, \mathcal{K}} \mathcal{P}(\varrho)\right)^{2} \\
& =\left(\mathfrak{J}^{\rho, \mathcal{K}} \Theta_{2}(\varrho)-\mathfrak{J}^{\rho, \mathcal{K}} \mathcal{P}(\varrho)\right)\left(\mathfrak{J}^{\rho, \mathcal{K}} \mathcal{P}(\varrho)-\mathfrak{J}^{\rho, \mathcal{K}} \Theta_{1}(\varrho)\right) \\
& \quad-\frac{\varrho^{\frac{\rho}{\mathcal{K}}}}{\Gamma_{\mathcal{K}}(\rho+\mathcal{K})}\left(\mathfrak{J}^{\rho, \mathcal{K}} \Theta_{2}(\varrho)-\mathfrak{J}^{\rho, \mathcal{K}} \mathcal{P}(\varrho)\right)\left(\mathfrak{J}^{\rho, \mathcal{K}} \mathcal{P}(\varrho)-\mathfrak{J}^{\rho, \mathcal{K}} \Theta_{1}(\varrho)\right) \\
& \quad+\frac{\varrho}{\Gamma_{\mathcal{K}}(\rho+\mathcal{K})}\left(\mathfrak{J}^{\rho, \mathcal{K}}\left(\Theta_{1}(\varrho) \mathcal{P}(\varrho)\right)-\mathfrak{J}^{\rho, \mathcal{K}} \Theta_{1}(\varrho) \mathfrak{J}^{\rho, \mathcal{K}} \mathcal{P}(\varrho)\right. \\
& \quad+\frac{\varrho \frac{\rho}{\mathcal{K}}}{\Gamma_{\mathcal{K}}(\rho+\mathcal{K})} \mathfrak{J}^{\rho, \mathcal{K}}\left(\Theta_{2}(\varrho) \mathcal{P}(\varrho)\right)-\mathfrak{J}^{\rho, \mathcal{K}} \Theta_{2}(\varrho) \mathfrak{J}^{\rho, \mathcal{K}} \mathcal{P}(\varrho) \\
& \quad+\mathfrak{J}^{\rho, \mathcal{K}}\left(\Theta_{1}(\varrho)\right) \mathfrak{J}^{\rho, \mathcal{K}}\left(\Theta_{2}(\varrho)\right)-\frac{\varrho^{\mathcal{K}}}{\Gamma_{\mathcal{K}}(\rho+\mathcal{K})} \mathfrak{J}^{\rho, \mathcal{K}}\left(\Theta_{1}(\varrho) \Theta_{2}(\varrho)\right) .
\end{aligned}
$$

Corollary 2.11 Let $m<\mathcal{M}, \mathcal{K}, \rho, \delta>0, \mathcal{P}$ be a positive function on $[0, \infty)$ such that $m \leq$ $\mathcal{P}(\varrho) \leq \mathcal{M}$, and $\Psi$ be a positive and increasing function on $[0, \infty)$ such that $\Psi(0)=0$ and 
$\Psi^{\prime}$ is continuous on $[0, \infty)$. Then

$$
\begin{aligned}
& \frac{\Psi \stackrel{\rho}{\mathcal{K}}(\varrho)}{\Gamma_{\mathcal{K}}(\rho+\mathcal{K})} \mathfrak{J}_{\Psi, 0^{+}}^{\rho, \mathcal{K}} \mathcal{P}^{2}(\varrho)-\left(\mathfrak{J}_{\Psi, 0^{+}}^{\rho, \mathcal{K}} \mathcal{P}(\varrho)\right)^{2} \\
& =\left(\mathcal{M} \frac{\Psi \stackrel{\rho}{\mathcal{K}}(\varrho)}{\Gamma_{\mathcal{K}}(\rho+\mathcal{K})}-\mathfrak{J}_{\Psi, 0^{+}}^{\rho, \mathcal{K}} \mathcal{P}(\varrho)\right)\left(\mathfrak{J}_{\Psi, 0^{+}}^{\rho, \mathcal{K}} \mathcal{P}(\varrho)-m \frac{\Psi^{\stackrel{\rho}{\mathcal{K}}}(\varrho)}{\Gamma_{\mathcal{K}}(\rho+\mathcal{K})}\right) \\
& \left.\quad-\frac{\Psi \frac{\rho}{\mathcal{K}}(\varrho)}{\Gamma_{\mathcal{K}}(\rho+\mathcal{K})} \mathfrak{J}_{\Psi, 0^{+}}^{\rho, \mathcal{K}}(\mathcal{M}-\mathcal{P}(\varrho))(\mathcal{P}(\varrho)-m)\right)
\end{aligned}
$$

Remark 2.12 From Lemma 2.9 and Corollary 2.11 we get three conclusions as follows.

(1) If $\mathcal{K}=1$, then Lemma 2.9 leads to Lemma 2.19 in [68].

(2) If $\mathcal{K}=1$ and $\Psi(\varrho)=\varrho$, then Lemma 2.9 becomes Lemma 7 in [69].

(3) If $\mathcal{K}=1$ and $\Psi(\varrho)=\varrho$, then Corollary 2.11 leads to Corollary 8 in [69].

Theorem 2.13 Let $\mathcal{K}, \varrho>0, \mathcal{P}, \mathcal{S}, \Theta_{1}, \Theta_{2}, \varphi_{1}$ and $\varphi_{2}$ be six integrable functions defined on $[0, \infty)$, and $\Psi$ be an increasing and positive function on $[0, \infty)$ such that $\Psi^{\prime}(x)$ is continuous on $[0, \infty)$ and $\Psi(0)=0$. If conditions (2.1) and (2.6) are satisfied, then one has

$$
\begin{aligned}
& \left|\frac{\Psi \frac{\rho}{\mathcal{K}}}{\Gamma_{\mathcal{K}}(\rho+\mathcal{K})} \mathfrak{J}_{\Psi, 0^{+}}^{\rho, \mathcal{K}}(\mathcal{P}(\varrho) \mathcal{S}(\varrho))-\mathfrak{J}_{\Psi, 0^{+}}^{\rho, \mathcal{K}} \mathcal{P}(\varrho) \mathfrak{J}_{\Psi, 0^{+}}^{\rho, \mathcal{K}} \mathcal{S}(\varrho)\right| \\
& \quad \leq \sqrt{\mathfrak{T}\left(\mathcal{P}, \Theta_{1}, \Theta_{2}\right) \mathfrak{T}\left(\mathcal{S}, \varphi_{1}, \varphi_{2}\right)}
\end{aligned}
$$

where

$$
\begin{aligned}
\mathfrak{T}\left(\mathcal{P}, \Theta_{1}, \Theta_{2}\right)= & \left(\mathfrak{J}_{\Psi, 0^{+}}^{\rho, \mathcal{K}} \Theta_{2}(\varrho)-\mathfrak{J}_{\Psi, 0^{+}}^{\rho, \mathcal{K}} \mathcal{P}(\varrho)\right)\left(\mathfrak{J}_{\Psi, 0^{+}}^{\rho, \mathcal{K}} \mathcal{P}(\varrho)-\mathfrak{J}_{\Psi, 0^{+}}^{\rho, \mathcal{K}} \Theta_{1}(\varrho)\right) \\
& +\frac{\Psi \frac{\rho}{\mathcal{K}}(\varrho)}{\Gamma_{\mathcal{K}}(\rho+\mathcal{K})} \mathfrak{J}_{\Psi, 0^{+}}^{\rho, \mathcal{K}}\left(\Theta_{1}(\varrho) \mathcal{P}(\varrho)\right)-\mathfrak{J}_{\Psi, 0^{+}}^{\rho, \mathcal{K}} \Theta_{1}(\varrho) \mathfrak{J}_{\Psi, 0^{+}}^{\rho, \mathcal{K}} \mathcal{P}(\varrho) \\
& +\frac{\Psi \stackrel{\rho}{\mathcal{K}}(\varrho)}{\Gamma_{\mathcal{K}}(\rho+\mathcal{K})} \mathfrak{J}_{\Psi, 0^{+}}^{\rho, \mathcal{K}} \Theta_{2}(\varrho) \mathcal{P}(\varrho) \\
& -\mathfrak{J}_{\Psi, 0^{+}}^{\rho, \mathcal{K}} \Theta_{2}(\varrho) \mathfrak{J}_{\Psi, 0^{+}}^{\rho, \mathcal{K}} \mathcal{P}(\varrho)+\mathfrak{J}_{\Psi, 0^{+}}^{\rho, \mathcal{K}} \Theta_{1}(\varrho) \mathfrak{J}_{\Psi, 0^{+}}^{\rho, \mathcal{K}} \Theta_{2}(\varrho) \\
& -\frac{\Psi \frac{\rho}{\mathcal{K}}(\varrho)}{\Gamma_{\mathcal{K}}(\rho+\mathcal{K})} \mathfrak{J}_{\Psi, 0^{+}}^{\rho, \mathcal{K}}\left(\Theta_{1}(\varrho) \Theta_{2}(\varrho)\right)
\end{aligned}
$$

and

$$
\begin{aligned}
\mathfrak{T}\left(\mathcal{S}, \varphi_{1}, \varphi_{2}\right)= & \left(\mathfrak{J}_{\Psi, 0^{+}}^{\rho, \mathcal{K}} \varphi_{2}(\varrho)-\mathfrak{J}_{\Psi, 0^{+}}^{\rho, \mathcal{K}} \mathcal{S}(\varrho)\right)\left(\mathfrak{J}_{\Psi, 0^{+}}^{\rho, \mathcal{K}} \mathcal{S}(\varrho)-\mathfrak{J}_{\Psi, 0^{+}}^{\rho, \mathcal{K}} \varphi_{1}(\varrho)\right) \\
& +\frac{\Psi^{\stackrel{\rho}{\mathcal{K}}}(\varrho)}{\Gamma_{\mathcal{K}}(\rho+\mathcal{K})} \mathfrak{J}_{\Psi, 0^{+}}^{\rho, \mathcal{K}}\left(\varphi_{1}(\varrho) \mathcal{S}(\varrho)\right)-\mathfrak{J}_{\Psi, 0^{+}}^{\rho, \mathcal{K}} \varphi_{1}(\varrho) \mathfrak{J}_{\Psi, 0^{+}}^{\rho, \mathcal{K}} \mathcal{S}(\varrho) \\
& +\frac{\Psi^{\rho} \mathcal{K}}{\Gamma_{\mathcal{K}}(\rho+\mathcal{K})} \mathfrak{J}_{\Psi, 0^{+}}^{\rho, \mathcal{K}}\left(\varphi_{2}(\varrho) \mathcal{S}(\varrho)\right) \\
& -\mathfrak{J}_{\Psi, 0^{+}}^{\rho, \mathcal{K}} \varphi_{2}(\varrho) \mathfrak{J}_{\Psi, 0^{+}}^{\rho, \mathcal{K}} \mathcal{S}(\varrho)+\mathfrak{J}_{\Psi, 0^{+}}^{\rho, \mathcal{K}} \varphi_{1}(\varrho) \mathfrak{J}_{\Psi, 0^{+}}^{\rho, \mathcal{K}} \varphi_{2}(\varrho) \\
& -\frac{\Psi^{\frac{\rho}{\mathcal{K}}}(\varrho)}{\Gamma_{\mathcal{K}}(\rho+\mathcal{K})} \mathfrak{J}_{\Psi, 0^{+}}^{\rho, \mathcal{K}}\left(\varphi_{1}(\varrho) \varphi_{2}(\varrho)\right) .
\end{aligned}
$$


Proof Let $\varrho>0, \mathfrak{d}_{1}, \mathfrak{d}_{2} \in(0, \varrho), \mathcal{P}$ and $\mathcal{S}$ be two positive functions defined on $[0, \infty)$ such that (2.1) and (2.6) are satisfied, and $\mathfrak{T}\left(\mathfrak{d}_{1}, \mathfrak{d}_{2}\right)$ be defined by

$$
\mathfrak{T}\left(\mathfrak{d}_{1}, \mathfrak{d}_{2}\right)=\left(\mathcal{P}\left(\mathfrak{d}_{1}\right)-\mathcal{P}\left(\mathfrak{d}_{2}\right)\right)\left(\mathcal{S}\left(\mathfrak{d}_{1}\right)-\mathcal{S}\left(\mathfrak{d}_{2}\right)\right)
$$

Taking product on both sides of $(2.18)$ by $\frac{\left.\left(\Psi(\varrho)-\Psi\left(\mathfrak{d}_{1}\right)\right) \rho^{\frac{\rho}{\mathcal{K}}-1} \Psi^{\prime}\left(\mathfrak{d}_{1}\right)\left(\Psi(\rho)-\Psi\left(\mathfrak{d}_{2}\right)\right)\right)^{\frac{\rho}{\mathcal{K}}-1} \Psi^{\prime}\left(\mathfrak{d}_{2}\right)}{2\left(\mathcal{K} \Gamma_{\mathcal{K}}(\rho)\right)^{2}}$ and integrating the resulting identity for the variable $\mathfrak{d}_{1}$ and $\mathfrak{d}_{2}$ from 0 to $\varrho$, we obtain

$$
\begin{aligned}
& \frac{1}{2\left(\mathcal{K} \Gamma_{\mathcal{K}}(\rho)\right)^{2}} \int_{0}^{\varrho} \int_{0}^{\varrho}\left(\Psi(\varrho)-\Psi\left(\mathfrak{d}_{1}\right)\right)^{\frac{\rho}{\mathcal{K}}}-1\left(\Psi(\varrho)-\Psi\left(\mathfrak{d}_{2}\right)\right)^{\frac{\rho}{\mathcal{K}}-1} \mathfrak{T}\left(\mathfrak{d}_{1}, \mathfrak{d}_{2}\right) \\
& \quad \times \Psi^{\prime}\left(\mathfrak{d}_{1}\right) \Psi^{\prime}\left(\mathfrak{d}_{2}\right) d \mathfrak{d}_{1} d \mathfrak{d}_{2} \\
& =\frac{\psi \frac{\rho}{\mathcal{K}}(\varrho)}{\Gamma_{\mathcal{K}}(\rho+\mathcal{K})} \mathfrak{J}_{\Psi, 0^{+}}^{\rho, \mathcal{K}}(\mathcal{P}(\varrho) \mathcal{S}(\varrho))-\mathfrak{J}_{\Psi, 0^{+}}^{\rho, \mathcal{K}}(\mathcal{P}(\varrho)) \mathfrak{J}_{\Psi, 0^{+}}^{\rho, \mathcal{K}}(\mathcal{S}(\varrho)) .
\end{aligned}
$$

Applying the Cauchy-Schwarz inequality to (2.19), we have

$$
\begin{aligned}
& \left(\frac{1}{2\left(\mathcal{K} \Gamma_{\mathcal{K}}(\rho)\right)^{2}} \int_{0}^{\varrho} \int_{0}^{\varrho}\left(\Psi(\varrho)-\Psi\left(\mathfrak{d}_{1}\right)\right)^{\frac{\rho}{\mathcal{K}}-1}\left(\Psi(\varrho)-\Psi\left(\mathfrak{d}_{2}\right)\right)^{\frac{\rho}{\mathcal{K}}-1}\left(\mathcal{P}\left(\mathfrak{d}_{1}\right)-\mathcal{P}\left(\mathfrak{d}_{2}\right)\right)\right. \\
& \left.\quad \times\left(\mathcal{S}\left(\mathfrak{d}_{1}\right)-\mathcal{S}\left(\mathfrak{d}_{2}\right)\right) \Psi^{\prime}\left(\mathfrak{d}_{1}\right) \Psi^{\prime}\left(\mathfrak{d}_{2}\right) d \mathfrak{d}_{1} d \mathfrak{d}_{2}\right)^{2} \\
& \leq \frac{1}{2\left(\mathcal{K} \Gamma_{\mathcal{K}}(\rho)\right)^{2}} \int_{0}^{\varrho} \int_{0}^{\varrho}\left(\Psi(\varrho)-\Psi\left(\mathfrak{d}_{1}\right)\right)^{\frac{\rho}{\mathcal{K}}-1}\left(\Psi(\varrho)-\Psi\left(\mathfrak{d}_{2}\right)\right)^{\frac{\rho}{\mathcal{K}}-1}\left(\mathcal{P}\left(\mathfrak{d}_{1}\right)-\mathcal{P}\left(\mathfrak{d}_{2}\right)\right)^{2} \\
& \quad \times \Psi^{\prime}\left(\mathfrak{d}_{1}\right) \Psi^{\prime}\left(\mathfrak{d}_{2}\right) d \mathfrak{d}_{1} d \mathfrak{d}_{2} \\
& \quad \times \frac{1}{2\left(\mathcal{K} \Gamma_{\mathcal{K}}(\rho)\right)^{2}} \int_{0}^{\varrho} \int_{0}^{\varrho}\left(\Psi(\varrho)-\Psi\left(\mathfrak{d}_{1}\right)\right)^{\frac{\rho}{\mathcal{K}}-1}\left(\Psi(\varrho)-\Psi\left(\mathfrak{d}_{2}\right)\right)^{\frac{\rho}{\mathcal{K}}-1}\left(\mathcal{S}\left(\mathfrak{d}_{1}\right)-\mathcal{S}\left(\mathfrak{d}_{2}\right)\right)^{2} \\
& \quad \times \Psi^{\prime}\left(\mathfrak{d}_{1}\right) \Psi^{\prime}\left(\mathfrak{d}_{2}\right) d \mathfrak{d}_{1} d \mathfrak{d}_{2} .
\end{aligned}
$$

From (2.19) and (2.20) we get

$$
\begin{aligned}
& \left(\frac{\psi^{\frac{\rho}{\mathcal{K}}}(\varrho)}{\Gamma_{\mathcal{K}}(\rho+\mathcal{K})} \mathfrak{J}_{\Psi, 0^{+}}^{\rho, \mathcal{K}}(\mathcal{P}(\varrho) \mathcal{S}(\varrho))-\mathfrak{J}_{\Psi, 0^{+}}^{\rho, \mathcal{K}}(\mathcal{P}(\varrho)) \mathfrak{J}_{\Psi, 0^{+}}^{\rho, \mathcal{K}}(\mathcal{S}(\varrho))\right)^{2} \\
& \leq\left(\frac{\Psi \frac{\rho}{\mathcal{K}}(\varrho)}{\Gamma_{\mathcal{K}}(\rho+\mathcal{K})} \mathfrak{J}_{\Psi, 0^{+}}^{\rho, \mathcal{K}} \mathcal{P}^{2}(\varrho)-\left(\mathfrak{J}_{\Psi, 0^{+}}^{\rho, \mathcal{K}} \mathcal{P}(\varrho)\right)^{2}\right) \\
& \quad \times\left(\frac{\Psi \frac{\rho}{\mathcal{K}}(\varrho)}{\Gamma_{\mathcal{K}}(\rho+\mathcal{K})} \mathfrak{J}_{\Psi, 0^{+}}^{\rho, \mathcal{K}} \mathcal{S}^{2}(\varrho)-\left(\mathfrak{J}_{\Psi, 0^{+}}^{\rho, \mathcal{K}} \mathcal{S}(\varrho)\right)^{2}\right)
\end{aligned}
$$

Since $\left(\Theta_{2}(\varrho)-\mathcal{P}(\varrho)\right)\left(\mathcal{P}(\varrho)-\Theta_{1}(\varrho)\right) \geq 0$ and $\left(\varphi_{2}(\varrho)-\mathcal{S}(\varrho)\right)\left(\mathcal{S}(\varrho)-\varphi_{1}(\varrho)\right) \geq 0$ for $\varrho \in[0, \infty)$, we have

$$
\begin{aligned}
& \frac{\Psi \frac{\rho}{\mathcal{K}}(\varrho)}{\Gamma_{\mathcal{K}}(\rho+\mathcal{K})} \mathfrak{J}_{\Psi, 0^{+}}^{\rho, \mathcal{K}}\left(\Theta_{2}(\varrho)-\mathcal{P}(\varrho)\right)\left(\mathcal{P}(\varrho)-\Theta_{1}(\varrho)\right) \geq 0, \\
& \frac{\Psi \frac{\rho}{\mathcal{K}}(\varrho)}{\Gamma_{\mathcal{K}}(\rho+\mathcal{K})} \mathfrak{J}_{\Psi, 0^{+}}^{\rho, \mathcal{K}}\left(\varphi_{2}(\varrho)-\mathcal{S}(\varrho)\right)\left(\mathcal{S}(\varrho)-\varphi_{1}(\varrho)\right) \geq 0 .
\end{aligned}
$$


Thus from Lemma 2.9 we obtain

$$
\begin{aligned}
& \left(\frac{\Psi \stackrel{\rho}{\mathcal{K}}(\varrho)}{\Gamma_{\mathcal{K}}(\rho+\mathcal{K})} \mathfrak{J}_{\Psi, 0^{+}}^{\rho, \mathcal{K}} \mathcal{P}^{2}(\varrho)-\left(\mathfrak{J}_{\Psi, 0^{+}}^{\rho, \mathcal{K}} \mathcal{P}(\varrho)\right)^{2}\right) \\
& \leq\left(\mathfrak{J}_{\Psi, 0^{+}}^{\rho, \mathcal{K}} \Theta_{2}(\varrho)-\mathfrak{J}_{\Psi, 0^{+}}^{\rho, \mathcal{K}} \mathcal{P}(\varrho)\right)\left(\mathfrak{J}_{\Psi, 0^{+}}^{\rho, \mathcal{K}} \mathcal{P}(\varrho)-\mathfrak{J}_{\Psi, 0^{+}}^{\rho, \mathcal{K}} \Theta_{1}(\varrho)\right) \\
& +\frac{\Psi \stackrel{\rho}{\mathcal{K}}(\varrho)}{\Gamma_{\mathcal{K}}(\rho+\mathcal{K})} \mathfrak{J}_{\Psi, 0^{+}}^{\rho, \mathcal{K}}\left(\Theta_{1}(\varrho) \mathcal{P}(\varrho)\right) \\
& -\mathfrak{J}_{\Psi, 0^{+}}^{\rho, \mathcal{K}} \Theta_{1}(\varrho) \mathfrak{J}_{\Psi, 0^{+}}^{\rho, \mathcal{K}} \mathcal{P}(\varrho)+\frac{\Psi^{\rho} \mathcal{K}}{\Gamma_{\mathcal{K}}(\rho+\mathcal{K})} \mathfrak{J}_{\Psi, 0^{+}}^{\rho, \mathcal{K}} \Theta_{2}(\varrho) \mathcal{P}(\varrho)-\mathfrak{J}_{\Psi, 0^{+}}^{\rho, \mathcal{K}} \Theta_{2}(\varrho) \mathfrak{J}_{\Psi, 0^{+}}^{\rho, \mathcal{K}} \mathcal{P}(\varrho) \\
& +\mathfrak{J}_{\Psi, 0^{+}}^{\rho, \mathcal{K}} \Theta_{1}(\varrho) \mathfrak{J}_{\Psi, 0^{+}}^{\rho, \mathcal{K}} \Theta_{2}(\varrho)-\frac{\Psi \stackrel{\rho}{\mathcal{K}}(\varrho)}{\Gamma_{\mathcal{K}}(\rho+\mathcal{K})} \mathfrak{J}_{\Psi, 0^{+}}^{\rho, \mathcal{K}}\left(\Theta_{1}(\varrho) \Theta_{2}(\varrho)\right) \\
& =\mathfrak{T}\left(\mathcal{P}, \Theta_{1}, \Theta_{2}\right), \\
& \left(\frac{\Psi \stackrel{\rho}{\mathcal{K}}(\varrho)}{\Gamma_{\mathcal{K}}(\rho+\mathcal{K})} \mathfrak{J}_{\Psi, 0^{+}}^{\rho, \mathcal{K}} \mathcal{S}^{2}(\varrho)-\left(\mathfrak{J}_{\Psi, 0^{+}}^{\rho, \mathcal{K}} \mathcal{S}(\varrho)\right)^{2}\right) \\
& \leq\left(\mathfrak{J}_{\Psi, 0^{+}}^{\rho, \mathcal{K}} \varphi_{2}(\varrho)-\mathfrak{J}_{\Psi, 0^{+}}^{\rho, \mathcal{K}} \mathcal{S}(\varrho)\right)\left(\mathfrak{J}_{\Psi, 0^{+}}^{\rho, \mathcal{K}} \mathcal{S}(\varrho)-\mathfrak{J}_{\Psi, 0^{+}}^{\rho, \mathcal{K}} \varphi_{1}(\varrho)\right) \\
& +\frac{\Psi \frac{\rho}{\mathcal{K}}(\varrho)}{\Gamma_{\mathcal{K}}(\rho+\mathcal{K})} \mathfrak{J}_{\Psi, 0^{+}}^{\rho, \mathcal{K}}\left(\varphi_{1}(\varrho) \mathcal{S}(\varrho)\right) \\
& -\mathfrak{J}_{\Psi, 0^{+}}^{\rho, \mathcal{K}} \varphi_{1}(\varrho) \mathfrak{J}_{\Psi, 0^{+}}^{\rho, \mathcal{K}} \mathcal{S}(\varrho)+\frac{\Psi \frac{\rho}{\mathcal{K}}(\varrho)}{\Gamma_{\mathcal{K}}(\rho+\mathcal{K})} \mathfrak{J}_{\Psi, 0^{+}}^{\rho, \mathcal{K}}\left(\varphi_{2}(\varrho) \mathcal{S}(\varrho)\right)-\mathfrak{J}_{\Psi, 0^{+}}^{\rho, \mathcal{K}} \varphi_{2}(\varrho) \mathfrak{J}_{\Psi, 0^{+}}^{\rho, \mathcal{K}} \mathcal{S}(\varrho) \\
& +\mathfrak{J}_{\Psi, 0^{+}}^{\rho, \mathcal{K}} \varphi_{1}(\varrho) \mathfrak{J}_{\Psi, 0^{+}}^{\rho, \mathcal{K}} \varphi_{2}(\varrho)-\frac{\Psi \stackrel{\rho}{\mathcal{K}}(\varrho)}{\Gamma_{\mathcal{K}}(\rho+\mathcal{K})} \mathfrak{J}_{\Psi, 0^{+}}^{\rho, \mathcal{K}}\left(\varphi_{1}(\varrho) \varphi_{2}(\varrho)\right) \\
& =\mathfrak{T}\left(\mathcal{S}, \varphi_{1}, \varphi_{2}\right)
\end{aligned}
$$

Therefore, inequality (2.17) follows from (2.20), (2.24), and (2.25).

Corollary 2.14 Let $m, M, n, N \in \mathcal{R}, \mathfrak{T}\left(\mathcal{P}, \Theta_{1}, \Theta_{2}\right)=\mathfrak{T}(\mathcal{P}, m, M)$ and $\mathfrak{T}\left(\mathcal{S}, \varphi_{1}, \varphi_{2}\right)=$ $\mathfrak{T}(\mathcal{S}, n, N)$. Then inequality $(2.17)$ reduces to

$$
\begin{aligned}
& \left|\frac{\Psi \stackrel{\rho}{\mathcal{K}}(\varrho)}{\Gamma_{\mathcal{K}}(\rho+\mathcal{K})} \mathfrak{J}_{\Psi, 0^{+}}^{\rho, \mathcal{K}}(\mathcal{P}(\varrho) \mathcal{S}(\varrho))-\mathfrak{J}_{\Psi, 0^{+}}^{\rho, \mathcal{K}} \mathcal{P}(\varrho) \mathfrak{J}_{\Psi, 0^{+}}^{\rho, \mathcal{K}} \mathcal{S}(\varrho)\right| \\
& \quad \leq\left(\frac{\Psi \stackrel{\rho}{\mathcal{K}}(\varrho)}{2 \Gamma_{\mathcal{K}}(\rho+\mathcal{K})}\right)^{2}(M-m)(N-n) .
\end{aligned}
$$

Corollary 2.15 Let $m, \mathcal{M}, n, \mathcal{N} \in \mathcal{R}, \quad \Psi(\varrho)=\varrho, \mathfrak{T}\left(\mathcal{P}, \Theta_{1}, \Theta_{2}\right)=\mathfrak{T}(\mathcal{P}, m, \mathcal{M})$ and $\mathfrak{T}\left(\mathcal{S}, \varphi_{1}, \varphi_{2}\right)=\mathfrak{T}(\mathcal{S}, n, \mathcal{N})$. Then inequality (2.17) leads to

$$
\begin{aligned}
& \left|\frac{\varrho^{\stackrel{\rho}{\mathcal{K}}}}{\Gamma_{\mathcal{K}}(\rho+\mathcal{K})} \mathfrak{J}^{\rho, \mathcal{K}}(\mathcal{P}(\varrho) \mathcal{S}(\varrho))-\mathfrak{J}^{\rho, \mathcal{K}} \mathcal{P}(\varrho) \mathfrak{J}^{\rho, \mathcal{K}} \mathcal{S}(\varrho)\right| \\
& \quad \leq\left(\frac{\varrho^{\rho} \mathcal{K}}{\Gamma_{\mathcal{K}}(\rho+\mathcal{K})}\right)^{2}(\mathcal{M}-m)(\mathcal{N}-n) .
\end{aligned}
$$

Remark 2.16 Theorem 2.13 and Corollary 2.14 lead to four conclusions as follows:

(1) If $\mathcal{K}=1$, then Theorem 2.13 leads to Theorem 2.23 in [68]. 
(2) If $\mathcal{K}=1$ and $\Psi(\varrho)=\varrho$, then Theorem 2.13 gives Theorem 9 in [69].

(3) If $\mathcal{K}=1$, then Corollary 2.14 leads to Corollary 2.26 in [68].

(4) If $\mathcal{K}=1$ and $\Psi(\varrho)=\varrho$, then Corollary 2.14 gives Remark 10 in [69].

Example 2.17 Let $\mathcal{K}, \rho, \delta>0, \mathcal{P}$ and $\mathcal{S}$ be two positive functions defined on $[0, \infty)$, and $\Psi$ be an increasing and positive function on $[0, \infty)$ such that $\Psi^{\prime}$ is continuous on $[0, \infty)$ with $\Psi(0)=0$. Then one has
(a) $\quad q \mathfrak{J}_{\Psi, 0^{+}}^{\rho, \mathcal{K}} \mathcal{P}^{p}(\varrho)+p \mathfrak{J}_{\Psi, 0^{+}}^{\rho, \mathcal{K}} \mathcal{S}^{q}(\varrho) \geq p q \frac{\Gamma_{\mathcal{K}}(\rho+\mathcal{K})}{\Psi \frac{\rho}{\mathcal{K}}(\varrho)}\left[\mathfrak{J}_{\Psi, 0^{+}}^{\rho, \mathcal{K}} \mathcal{S}(\varrho) \mathfrak{J}_{\Psi, 0^{+}}^{\rho, \mathcal{K}} \mathcal{P}(\varrho)\right]$,
(b) $\quad q \mathfrak{J}_{\Psi, 0^{+}}^{\rho, \mathcal{K}} \mathcal{P}^{p}(\varrho) \mathfrak{J}_{\Psi, 0^{+}}^{\rho, \mathcal{K}} \mathcal{S}^{p}(\varrho)+p \mathfrak{J}_{\Psi, 0^{+}}^{\rho, \mathcal{K}} \mathcal{P}^{q}(\varrho) \mathfrak{J}_{\Psi, 0^{+}}^{\rho, \mathcal{K}} \mathcal{S}^{q}(\varrho) \geq p q\left(\mathfrak{J}_{\Psi, 0^{+}}^{\rho, \mathcal{K}} \mathcal{P}(\varrho) \mathcal{S}(\varrho)\right)^{2}$
(c) $\quad q \mathfrak{J}_{\Psi, 0^{+}}^{\rho, \mathcal{K}} \mathcal{P}^{p}(\varrho) \mathfrak{J}_{\Psi, 0^{+}}^{\rho, \mathcal{K}} \mathcal{S}^{q}(\varrho)+p \mathfrak{J}_{\Psi, 0^{+}}^{\rho, \mathcal{K}} \mathcal{P}^{q}(\varrho) \mathfrak{J}_{\Psi, 0^{+}}^{\rho, \mathcal{K}} \mathcal{S}^{p}(\varrho)$

$$
\geq p q \mathfrak{J}_{\Psi, 0^{+}}^{\rho, \mathcal{K}} \mathcal{P}(\varrho) \mathcal{S}^{p-1}(\varrho) \mathfrak{J}_{\Psi, 0^{+}}^{\rho, \mathcal{K}} \mathcal{P} \mathcal{S}^{q-1}(\varrho)
$$
(d) $\quad q \mathfrak{J}_{\Psi, 0^{+}}^{\rho, \mathcal{K}} \mathcal{P}^{p}(\varrho) \mathfrak{J}_{\Psi, 0^{+}}^{\rho, \mathcal{K}} \mathcal{S}^{q}(\varrho)+p \mathfrak{J}_{\Psi, 0^{+}}^{\rho, \mathcal{K}} \mathcal{P}^{p}(\varrho) \mathfrak{J}_{\Psi, 0^{+}}^{\rho, \mathcal{K}} \mathcal{S}^{q}(\varrho)$
$\geq p q \tilde{J}_{\Psi, 0^{+}}^{\rho, \mathcal{K}} \mathcal{P}^{p-1}(\varrho) \mathcal{S}^{q-1}(\varrho) \mathfrak{J}_{\Psi, 0^{+}}^{\rho, \mathcal{K}} \mathcal{P} \mathcal{S}(\varrho)$,
(e) $\quad q \mathfrak{J}_{\Psi, 0^{+}}^{\rho, \mathcal{K}} \mathcal{P}^{p}(\varrho) \mathfrak{J}_{\Psi, 0^{+}}^{\rho, \mathcal{K}} \mathcal{S}^{2}(\varrho)+p \mathfrak{J}_{\Psi, 0^{+}}^{\rho, \mathcal{K}} \mathcal{P}^{2}(\varrho) \mathfrak{J}_{\Psi, 0^{+}}^{\rho, \mathcal{K}} \mathcal{S}^{q}(\varrho)$
$\geq p q \mathfrak{J}_{\Psi, 0^{+}}^{\rho, \mathcal{K}} \mathcal{P}(\varrho) \mathcal{S}(\varrho) \mathfrak{J}_{\Psi, 0^{+}}^{\rho, \mathcal{K}} \mathcal{P}^{\frac{2}{q}} \mathcal{S}^{\frac{2}{p}}(\varrho)$,
(f) $\quad q \mathfrak{J}_{\Psi, 0^{+}}^{\rho, \mathcal{K}} \mathcal{P}^{2}(\varrho) \mathfrak{J}_{\Psi, 0^{+}}^{\rho, \mathcal{K}} \mathcal{S}^{q}(\varrho)+p \mathfrak{J}_{\Psi, 0^{+}}^{\rho, \mathcal{K}} \mathcal{P}^{p}(\varrho) \mathfrak{J}_{\Psi, 0^{+}}^{\rho, \mathcal{K}} \mathcal{S}^{2}(\varrho)$

$$
\geq p q \mathfrak{J}_{\Psi, 0^{+}}^{\rho, \mathcal{K}} \mathcal{P}^{\frac{2}{p}}(\varrho) \mathcal{S}^{\frac{2}{q}}(\varrho) \mathfrak{J}_{\Psi, 0^{+}}^{\rho, \mathcal{K}} \mathcal{P}^{p-1} \mathcal{S}^{q-1}(\varrho),
$$
(g) $\quad q \mathfrak{J}_{\Psi, 0^{+}}^{\rho, \mathcal{K}} \mathcal{P}^{2} \mathcal{S}^{q}(\varrho)+p \mathfrak{J}_{\Psi, 0^{+}}^{\rho, \mathcal{K}} \mathcal{P}^{2}(\varrho) \mathcal{S}^{p}(\varrho)$

$$
\geq p q \frac{\Gamma_{\mathcal{K}}(\rho+\mathcal{K})}{\Psi \frac{\rho}{\mathcal{K}}(\varrho)} \mathfrak{J}_{\Psi, 0^{+}}^{\rho, \mathcal{K}} \mathcal{P}^{\frac{2}{p}}(\varrho) \mathcal{S}^{q-1}(\varrho) \mathfrak{J}_{\Psi, 0^{+}}^{\rho, \mathcal{K}} \mathcal{P}^{\frac{2}{q}}(\varrho) \mathcal{S}^{p-1}(\varrho)
$$

Proof According to the well-known Young inequality [70]

$$
\frac{1}{p} a^{p}+\frac{1}{q} b^{q} \geq a b \quad\left(a, b \geq 0, p, q>1, \frac{1}{p}+\frac{1}{q}=1\right)
$$

and putting $a=\mathcal{P}\left(\mathfrak{d}_{1}\right)$ and $b=\mathcal{S}\left(\mathfrak{d}_{2}\right)$, we have

$$
\frac{1}{p} \mathcal{P}^{p}\left(\mathfrak{d}_{1}\right)+\frac{1}{q} \mathcal{S}^{q}\left(\mathfrak{d}_{2}\right) \geq \mathcal{P}\left(\mathfrak{d}_{1}\right) \mathcal{S}\left(\mathfrak{d}_{2}\right)
$$

for all $\mathcal{P}\left(\mathfrak{d}_{1}\right), \mathcal{S}\left(\mathfrak{d}_{2}\right) \geq 0$.

Taking product on both sides of $(2.27)$ by $\frac{1}{\mathcal{K} \Gamma_{\mathcal{K}}(\rho)}\left(\Psi(\varrho)-\Psi\left(\mathfrak{d}_{1}\right)\right)^{\frac{\rho}{\mathcal{K}}}-1 \Psi^{\prime}\left(\mathfrak{d}_{1}\right)$ (which is positive due to $\left.\varrho \in\left(0, \mathfrak{d}_{1}\right)\right)$ and integrating the resulting identity for the variable $\mathfrak{d}_{1}$ from 0 to $\varrho$, we get

$$
\begin{aligned}
& \frac{1}{p} \frac{1}{\mathcal{K} \Gamma_{\mathcal{K}}(\rho)} \int_{0}^{\varrho}\left(\Psi(\varrho)-\Psi\left(\mathfrak{d}_{1}\right)\right)^{\frac{\rho}{\mathcal{K}}-1} \Psi^{\prime}\left(\mathfrak{d}_{1}\right) \mathcal{P}^{p}\left(\mathfrak{d}_{1}\right) d \mathfrak{d}_{1} \\
& \quad+\frac{1}{q} \mathcal{S}^{q}\left(\mathfrak{d}_{2}\right) \frac{1}{\mathcal{K} \Gamma_{\mathcal{K}}(\rho)} \int_{0}^{\varrho}\left(\Psi(\varrho)-\Psi\left(\mathfrak{d}_{1}\right)\right)^{\frac{\rho}{\mathcal{K}}-1} \Psi^{\prime}\left(\mathfrak{d}_{1}\right) d \mathfrak{d}_{1} \\
& \geq \mathcal{S}\left(\mathfrak{d}_{2}\right) \frac{1}{\mathcal{K} \Gamma_{\mathcal{K}}(\rho)} \int_{0}^{\varrho}\left(\Psi(\varrho)-\Psi\left(\mathfrak{d}_{1}\right)\right)^{\frac{\rho}{\mathcal{K}}-1} \Psi^{\prime}\left(\mathfrak{d}_{1}\right) \mathcal{P}\left(\mathfrak{d}_{1}\right) d \mathfrak{d}_{1}
\end{aligned}
$$


and

$$
\frac{1}{p} \mathfrak{J}_{\Psi, 0^{+}}^{\rho, \mathcal{K}} \mathcal{P}^{p}(\varrho)+\frac{1}{q} \mathcal{S}^{q}\left(\mathfrak{d}_{2}\right) \frac{\Psi \mathcal{K}(\varrho)}{\Gamma_{\mathcal{K}}(\rho+\mathcal{K})} \geq \mathcal{S}\left(\mathfrak{d}_{2}\right) \mathfrak{J}_{\Psi, 0^{+}}^{\rho, \mathcal{K}} \mathcal{P}(\varrho)
$$

Again, taking product on both sides of (2.29) by $\left.\frac{1}{\mathcal{K} \Gamma_{\mathcal{K}}(\rho)}\left(\Psi(\varrho)-\Psi\left(\mathfrak{d}_{2}\right)\right)\right)^{\frac{\rho}{K}-1} \Psi^{\prime}\left(\mathfrak{d}_{2}\right)$ and integrating the resulting identity for the variable $\mathfrak{d}_{2}$ from 0 to $\varrho$, we get

$$
\frac{1}{p} \mathfrak{J}_{\Psi, 0^{+}}^{\rho, \mathcal{K}} \mathcal{P}^{p}(\varrho) \frac{\Psi \frac{\rho}{\mathcal{K}}(\varrho)}{\Gamma_{\mathcal{K}}(\rho+\mathcal{K})}+\frac{1}{q} \mathfrak{J}_{\Psi, 0^{+}}^{\rho, \mathcal{K}} \mathcal{S}^{q}(\varrho) \frac{\Psi \stackrel{\rho}{\mathcal{K}}(\varrho)}{\Gamma_{\mathcal{K}}(\rho+\mathcal{K})} \geq \mathfrak{J}_{\Psi, 0^{+}}^{\rho, \mathcal{K}} \mathcal{S}(\varrho) \mathfrak{J}_{\Psi, 0^{+}}^{\rho, \mathcal{K}} \mathcal{P}(\varrho)
$$

and

$$
\frac{1}{p} \mathfrak{J}_{\Psi, 0^{+}}^{\rho, \mathcal{K}} \mathcal{P}^{p}(\varrho)+\frac{1}{q} \mathfrak{J}_{\Psi, 0^{+}}^{\rho, \mathcal{K}} \mathcal{S}^{q}(\varrho) \geq \frac{\Gamma_{\mathcal{K}}(\rho+\mathcal{K})}{\Psi \frac{\rho}{\mathcal{K}}(\varrho)}\left[\mathfrak{J}_{\Psi, 0^{+}}^{\rho, \mathcal{K}} \mathcal{S}(\varrho) \mathfrak{J}_{\Psi, 0^{+}}^{\rho, \mathcal{K}} \mathcal{P}(\varrho)\right]
$$

which implies part (a). The rest of inequalities can be shown in a similar manner by using the subsequent desire of parameters in the Young inequality:

(b) $\quad a=\mathcal{P}\left(\mathfrak{d}_{1}\right) \mathcal{S}\left(\mathfrak{d}_{2}\right), \quad b=\mathcal{P}\left(\mathfrak{d}_{2}\right) \mathcal{S}\left(\mathfrak{d}_{1}\right)$.

(c) $\quad a=\frac{\mathcal{P}\left(\mathfrak{d}_{1}\right)}{\mathcal{S}\left(\mathfrak{d}_{1}\right)}, \quad b=\frac{\mathcal{P}\left(\mathfrak{d}_{2}\right)}{\mathcal{S}\left(\mathfrak{d}_{2}\right)}, \quad \mathcal{S}\left(\mathfrak{d}_{1}\right) \mathcal{S}\left(\mathfrak{d}_{2}\right) \neq 0$.

(d) $\quad a=\frac{\mathcal{P}\left(\mathfrak{d}_{2}\right)}{\mathcal{P}\left(\mathfrak{d}_{1}\right)}, \quad b=\frac{\mathcal{S}\left(\mathfrak{d}_{2}\right)}{\mathcal{S}\left(\mathfrak{d}_{1}\right)}, \quad \mathcal{P}\left(\mathfrak{d}_{1}\right) \mathcal{S}\left(\mathfrak{d}_{2}\right) \neq 0$.

(e) $\quad a=\mathcal{P}\left(\mathfrak{d}_{1}\right) \mathcal{S}^{\frac{2}{p}}\left(\mathfrak{d}_{2}\right), \quad b=\mathcal{P}^{\frac{2}{q}}\left(\mathfrak{d}_{2}\right) \mathcal{S}\left(\mathfrak{d}_{1}\right)$.

(f) $\quad a=\frac{\mathcal{P}^{\frac{2}{p}}\left(\mathfrak{d}_{1}\right)}{\mathcal{P}\left(\mathfrak{d}_{2}\right)}, \quad b=\frac{\mathcal{S}^{\frac{2}{q}}\left(\mathfrak{d}_{1}\right)}{\mathcal{S}\left(\mathfrak{d}_{2}\right)}, \quad \mathcal{P}\left(\mathfrak{d}_{2}\right) \mathcal{S}\left(\mathfrak{d}_{2}\right) \neq 0$.

(g) $\quad a=\frac{\mathcal{P}^{\frac{2}{p}}\left(\mathfrak{d}_{1}\right)}{\mathcal{S}\left(\mathfrak{d}_{2}\right)}, \quad b=\frac{\mathcal{P}^{\frac{2}{q}}\left(\mathfrak{d}_{2}\right)}{\mathcal{S}\left(\mathfrak{d}_{1}\right)}, \quad \mathcal{S}\left(\mathfrak{d}_{1}\right) \mathcal{S}\left(\mathfrak{d}_{2}\right) \neq 0$.

Repeating the foregoing argument, we can obtain parts (b)-(g).

Example 2.18 Let $\mathcal{K}, \rho, \delta>0, \mathcal{P}$ and $\mathcal{S}$ be two positive functions defined on $[0, \infty), \Psi$ be an increasing and positive function on $[0, \infty)$ such that $\Psi^{\prime}$ is continuous on $[0, \infty)$ and $\Psi(0)=0$, and $m$ and $\mathcal{M}$ be defined by

$$
m=\min _{0 \leq \mathfrak{d}_{1} \leq \varrho} \frac{\mathcal{P}\left(\mathfrak{d}_{1}\right)}{\mathcal{S}\left(\mathfrak{d}_{1}\right)}, \quad \mathcal{M}=\max _{0 \leq \mathfrak{d}_{1} \leq \varrho} \frac{\mathcal{P}\left(\mathfrak{d}_{1}\right)}{\mathcal{S}\left(\mathfrak{d}_{1}\right)},
$$

respectively. Then we have

$$
\text { (a) } \begin{aligned}
0 & \leq \mathfrak{J}_{\Psi, 0^{+}}^{\rho, \mathcal{K}} \mathcal{P}^{2}(\varrho) \mathfrak{J}_{\Psi, 0^{+}}^{\rho, \mathcal{K}} \mathcal{S}^{2}(\varrho) \leq \frac{(m+\mathcal{M})^{2}}{4 m \mathcal{M}}\left(\mathfrak{J}_{\Psi, 0^{+}}^{\rho, \mathcal{K}} \mathcal{P} \mathcal{S}(\varrho)\right)^{2} \\
\text { (b) } \quad 0 & \leq \sqrt{\mathfrak{J}_{\Psi, 0^{+}}^{\rho, \mathcal{K}} \mathcal{P}^{2}(\varrho) \mathfrak{J}_{\Psi, 0^{+}}^{\rho, \mathcal{K}} \mathcal{S}^{2}(\varrho)}-\left(\mathfrak{J}_{\Psi, 0^{+}}^{\rho, \mathcal{K}} \mathcal{P}(\varrho) \mathcal{S}(\varrho)\right) \\
& \leq \frac{(\sqrt{\mathcal{M}}-\sqrt{m})^{2}}{2 \sqrt{m \mathcal{M}}} \mathfrak{J}_{\Psi, 0^{+}}^{\rho, \mathcal{K}} \mathcal{P}(\varrho) \mathcal{S}(\varrho)
\end{aligned}
$$


Proof It follows from (2.32) and

$$
\left(\frac{\mathcal{P}\left(\mathfrak{d}_{1}\right)}{\mathcal{S}\left(\mathfrak{d}_{1}\right)}-m\right)\left(\mathcal{M}-\frac{\mathcal{P}\left(\mathfrak{d}_{1}\right)}{\mathcal{S}\left(\mathfrak{d}_{1}\right)}\right) \mathcal{S}^{2}\left(\mathfrak{d}_{1}\right) \geq 0 \quad\left(0 \leq \varrho \leq \mathfrak{d}_{1}\right)
$$

that

$$
\mathcal{P}^{2}\left(\mathfrak{d}_{1}\right)+m \mathcal{M} \mathcal{S}^{2}\left(\mathfrak{d}_{1}\right) \leq(m+\mathcal{M}) \mathcal{P}\left(\mathfrak{d}_{1}\right) \mathcal{S}\left(\mathfrak{d}_{1}\right)
$$

Multiplying (2.33) by $\frac{1}{\mathcal{K} \Gamma_{\mathcal{K}}(\rho)}\left(\Psi(\varrho)-\Psi\left(\mathfrak{d}_{1}\right)\right)^{\frac{\rho}{\mathcal{K}}-1} \Psi^{\prime}\left(\mathfrak{d}_{1}\right)$ and integrating with respect to $\mathfrak{d}_{1}$ over $(0, \varrho)$, we get

$$
\begin{aligned}
& \frac{1}{\mathcal{K} \Gamma_{\mathcal{K}}(\rho)} \int_{0}^{\varrho}\left(\Psi(\varrho)-\Psi\left(\mathfrak{d}_{1}\right)\right)^{\frac{\rho}{\mathcal{K}}-1} \Psi^{\prime}\left(\mathfrak{d}_{1}\right) \mathcal{P}^{2}\left(\mathfrak{d}_{1}\right) d \mathfrak{d}_{1} \\
& \quad+m \mathcal{M} \frac{1}{\mathcal{K} \Gamma_{\mathcal{K}}(\rho)} \int_{0}^{\varrho}\left(\Psi(\varrho)-\Psi\left(\mathfrak{d}_{1}\right)\right)^{\frac{\rho}{\mathcal{K}}-1} \Psi^{\prime}\left(\mathfrak{d}_{1}\right) \mathcal{S}^{2}\left(\mathfrak{d}_{1}\right) d \mathfrak{d}_{1} \\
& \leq \frac{m+\mathcal{M}}{\mathcal{K} \Gamma_{\mathcal{K}}(\rho)} \int_{0}^{\varrho}\left(\Psi(\varrho)-\Psi\left(\mathfrak{d}_{1}\right)\right)^{\frac{\rho}{\mathcal{K}}-1} \Psi^{\prime}\left(\mathfrak{d}_{1}\right) \mathcal{P}\left(\mathfrak{d}_{1}\right) \mathcal{S}\left(\mathfrak{d}_{1}\right) d \mathfrak{d}_{1},
\end{aligned}
$$

which implies that

$$
\mathfrak{J}_{\Psi, 0^{+}}^{\rho, \mathcal{K}} \mathcal{P}^{2}(\varrho)+m \mathcal{M} \mathfrak{J}_{\Psi, 0^{+}}^{\rho, \mathcal{K}} \mathcal{S}^{2}(\varrho) \leq(m+\mathcal{M}) \mathfrak{J}_{\Psi, 0^{+}}^{\rho, \mathcal{K}} \mathcal{P}(\varrho) \mathcal{S}(\varrho)
$$

Alternately, it follows from $m, \mathcal{M}>0$ and

$$
\left(\sqrt{\mathfrak{J}_{\Psi, 0^{+}}^{\rho, \mathcal{K}} \mathcal{P}^{2}(\varrho)}-\sqrt{m \mathcal{M} \mathfrak{J}_{\Psi, 0^{+}}^{\rho, \mathcal{K}} \mathcal{S}^{2}(\varrho)}\right)^{2} \geq 0
$$

that

$$
2 \sqrt{\mathfrak{J}_{\Psi, 0^{+}}^{\rho, \mathcal{K}} \mathcal{P}^{2}(\varrho)} \sqrt{m \mathcal{M} \mathfrak{J}_{\Psi, 0^{+}}^{\rho, \mathcal{K}} \mathcal{S}^{2}(\varrho)} \leq \mathfrak{J}_{\Psi, 0^{+}}^{\rho, \mathcal{K}} \mathcal{P}^{2}(\varrho)+m \mathcal{M} \mathfrak{J}_{\Psi, 0^{+}}^{\rho, \mathcal{K}} \mathcal{S}^{2}(\varrho)
$$

Therefore,

$$
4 m \mathcal{M} \mathfrak{J}_{\Psi, 0^{+}}^{\rho, \mathcal{K}} \mathcal{P}^{2}(\varrho) \mathfrak{J}_{\Psi, 0^{+}}^{\rho, \mathcal{K}} \mathcal{S}^{2}(\varrho) \leq(m+\mathcal{M})^{2}\left(\mathfrak{J}_{\Psi, 0^{+}}^{\rho, \mathcal{K}} \mathcal{P}(\varrho) \mathcal{S}(\varrho)\right)^{2}
$$

follows from (2.34) and (2.35), and part (a) is proved. By using a few transformations to part (a), we can obtain part (b).

\section{Conclusion}

This article begins with a succinct review of fractional integrals in the frame of a new fractional integral operator. We characterize the definition of generalized $\mathcal{K}$-fractional integral operators. We modify the Grüss type inequality by employing generalized $\mathcal{K}$-fractional integrals; specifically, the variant involving fractional integrals in the generalized RiemannLiouville and $\mathcal{K}$-fractional integrals frame is provided. The associated significant variants including summed up generalized $\mathcal{K}$-fractional integrals are also outlined. Numerous consequences can be generalized for the utility of these recently presented fractional integral operators. 


\section{Acknowledgements}

The authors would like to express their sincere thanks to the editor and anonymous reviewers for their helpful comments and suggestions.

\section{Funding}

No funding.

\section{Availability of data and materials}

Not applicable.

\section{Competing interests}

The authors declare that they have no competing interests.

\section{Authors' contributions}

All authors contributed equally to the writing of this paper. All authors read and approved the final manuscript.

\section{Author details}

'Department of Mathematics, Government College University, Faisalabad, Pakistan. ${ }^{2}$ Department of Mathematics, Cankaya University, Ankara, Turkey. ${ }^{3}$ Department of Mathematics, COMSATS University Islamabad, Islamabad, Pakistan. ${ }^{4}$ Institute of Space Sciences, Magurele-Bucharest, Romania. ${ }^{5}$ Department of Medical Research, China Medical University Hospital, China Medical University, Taichung, Taiwan. ${ }^{6}$ School of Mathematics and Physics, Anhui Jianzhu University, Hefei, China. ${ }^{7}$ Department of Mathematics, Southeast University, Nanjing, China.

\section{Publisher's Note}

Springer Nature remains neutral with regard to jurisdictional claims in published maps and institutional affiliations.

Received: 16 August 2019 Accepted: 16 April 2020 Published online: 11 May 2020

\section{References}

1. Huang, C.-X., Liu, L.-Z.: Sharp function inequalities and boundedness for Toeplitz type operator related to general fractional singular integral operator. Publ. Inst. Math. 92(106), 165-176 (2012)

2. Khalil, R., Al Horani, M., Yousef, A., Sababheh, M.: A new definition of fractional derivative. J. Comput. Appl. Math. 264, 65-70 (2014)

3. Adjabi, Y., Jarad, F., Baleanu, D., Abdeljawad, T.: On Cauchy problems with Caputo Hadamard fractional derivatives. J. Comput. Anal. Appl. 21(4), 661-681 (2016)

4. Nisar, K.S., Rahman, G., Baleanu, D., Mubeen, S., Arshad, M.: The (k, s)-fractional calculus of k-Mittag-Leffler function. Adv. Differ. Equ. 2017, Article ID 118 (2017)

5. Adil Khan, M., Begum, S., Khurshid, Y., Chu, Y.-M.: Ostrowski type inequalities involving conformable fractional integrals. J. Inequal. Appl. 2018, Article ID 70 (2018)

6. Adil Khan, M., Chu, Y.-M., Kashuri, A., Liko, R., Ali, G.: Conformable fractional integrals versions of Hermite-Hadamard inequalities and their generalizations. J. Funct. Spaces 2018, Article ID 6928130 (2018)

7. Adil Khan, M., Iqbal, A., Suleman, M., Chu, Y.-M.: Hermite-Hadamard type inequalities for fractional integrals via Green's function. J. Inequal. Appl. 2018, Article ID 161 (2018)

8. Adil Khan, M., Khurshid, Y., Du, T.-S., Chu, Y.-M.: Generalization of Hermite-Hadamard type inequalities via conformable fractional integrals. J. Funct. Spaces 2018, Article ID 5357463 (2018)

9. Khurshid, Y., Adil Khan, M., Chu, Y.-M., Khan, Z.A.: Hermite-Hadamard-Fejér inequalities for conformable fractional integrals via preinvex functions. J. Funct. Spaces 2019, Article ID 3146210 (2019)

10. Baleanu, D., Diethelm, K., Scalas, E., Trujillo, J.J.: Fractional Calculus: Models and Numerical Methods. World Scientific Hackensack (2012)

11. Kilbas, A.A., Srivastava, H.M., Trujillo, J.J.: Theory and Applications of Fractional Differential Equations. North-Holland Mathematics Studies, vol. 204. Elsevier, Amsterdam (2006)

12. Mainardi, F: Fractional Calculus and Waves in Linear Viscoelasticity. Imperial College Press, London (2010)

13. Akman, T., Yıldız, B., Baleanu, D.: New discretization of Caputo-Fabrizio derivative. Comput. Appl. Math. 37(3), 3307-3333 (2018)

14. Oliveira, D.S., Capelas de Oliveira, E.: Hilfer-Katugampola fractional derivative (2017, submitted). arXiv:1705.07733

15. Katugampola, U.N.: New approach to generalized fractional integral. Appl. Math. Comput. 218, 860-865 (2011)

16. Jarad, F., Abdeljawad, T., Baleanu, B.: On the generalized fractional derivatives and their Caputo modification. J. Nonlinear Sci. Appl. 10(5), 2607-2619 (2017)

17. Jarad, F., Abdeljawad, T.: Generalized fractional derivatives and Laplace transform. Discrete Contin. Dyn. Syst., Ser. S 13(3), 709-722 (2020). https://doi.org/10.3934/dcdss.2020039

18. Mubeen, S., Habibullah, G.M.: k-Fractional integrals and application. Int. J. Contemp. Math. Sci. 7(2), 89-94 (2012)

19. Azam, M.K., Farid, G., Rehman, M.A.: Study of generalized type $\kappa$-fractional derivatives. Adv. Differ. Equ. 2017, Article ID $249(2017)$

20. Set, E., Tomar, M., Sarikaya, M.Z.: On generalized Grüss type inequality for $\boldsymbol{\kappa}$-fractional integrals. Appl. Math. Comput. 269, 29-34 (2015)

21. Chu, Y.-M., Wang, M.-K., Qiu, S.-L.: Optimal combinations bounds of root-square and arithmetic means for Toader mean. Proc. Indian Acad. Sci. Math. Sci. 122(1), 41-51 (2012)

22. Wang, M.-K., Chu, Y.-M., Jiang, Y.-P.: Ramanujan's cubic transformation inequalities for zero-balanced hypergeometric functions. Rocky Mt. J. Math. 46(2), 670-691 (2016)

23. Qian, W.-M., Zhang, X.-H., Chu, Y.-M.: Sharp bounds for the Toader-Qi mean in terms of harmonic and geometric means. J. Math. Inequal. 11(1), 121-127 (2017) 
24. He, X.-H., Qian, W.-M., Xu, H.-Z., Chu, Y.-M.: Sharp power mean bounds for two Sándor-Yang means. Rev. R. Acad Cienc. Exactas Fís. Nat., Ser. A Mat. 113(3), 2627-2638 (2019)

25. Adil Khan, M., Hanif, M., Khan, Z.A., Ahmad, K., Chu, Y.-M.: Association of Jensen's inequality for s-convex function with Csiszár divergence. J. Inequal. Appl. 2019, Article ID 162 (2019)

26. Qian, W.-M., He, Z.-Y., Zhang, H.-W., Chu, Y.-M.: Sharp bounds for Neumann means in terms of two-parameter contraharmonic and arithmetic mean. J. Inequal. Appl. 2019. Article ID 168 (2019)

27. Wang, M.-K., Chu, Y.-M., Zhang, W.: Precise estimates for the solution of Ramanujan's generalized modular equation. Ramanujan J. 49(3), 653-668 (2019)

28. Wang, M.-K., Chu, H.-H., Chu, Y.-M.: Precise bounds for the weighted Hölder mean of the complete $p$-elliptic integrals. J. Math. Anal. Appl. 480(2), Article ID 123388 (2019). https://doi.org/10.1016/j.jmaa.2019.123388

29. Wang, M.-K., Zhang, W., Chu, Y.-M.: Monotonicity, convexity and inequalities involving the generalized elliptic integrals. Acta Math. Sci. 39B(5), 1440-1450 (2019)

30. Huang, C.-X., Guo, S., Liu, L.-Z.: Boundedness on Morrey space for Toeplitz type operator associated to singular integral operator with variable Calderón-Zygmund kernel. J. Math. Inequal. 8(3), 453-464 (2014)

31. Xie, D.-X., Li, J.: A new analysis of electrostatic free energy minimization and Poisson-Boltzmann equation for protein in ionic solvent. Nonlinear Anal., Real World Appl. 21, 185-196 (2015)

32. Zhou, W.-J., Wang, F.: A PRP-based residual method for large-scale monotone nonlinear equations. Appl. Math. Comput. 261, 1-7 (2015)

33. Wang, J.-F., Chen, X.-Y., Huang, L.-H.: The number and stability of limit cycles for planar piecewise linear systems of node-saddle type. J. Math. Anal. Appl. 469(1), 405-427 (2019)

34. Rashid, S., Akdemir, A.O., Jarad, F., Noor, M.A., Noor, K.I.: Simpson's type integral inequalities for $k$-fractional integrals and their applications. AIMS Math. 4(4), 1087-1100 (2019). https://doi.org/10.3934/math.2019.4.1087

35. Rashid, S., Noor, M.A., Noor, K.I.: Fractional exponentially $m$-convex functions and inequalities. Int. J. Anal. Appl. 17(3), 464-478 (2019). https://doi.org/10.28924/2291-8639

36. Rashid, S., Noor, M.A., Noor, K.I.: Some generalize Riemann-Liouville fractional estimates involving functions having exponentially convexity property. Punjab Univ. J. Math. 51(11), Article ID 01 (2019)

37. Rashid, S., Noor, M.A., Noor, K.I.: Inequalities pertaining fractional approach through exponentially convex functions. Fractal Fract. 3, Article ID 37 (2019). https://doi.org/10.3390/fractalfract3030037

38. Rashid, S., Noor, M.A., Noor, K.I.: New estimates for exponentially convex functions via conformable fractional operator. Fractal Fract. 3, Article ID 19 (2019). https://doi.org/10.3390/fractalfract3020019

39. Rashid, S., Noor, M.A., Noor, K.I., Safdar, F.: Integral inequalities for generalized preinvex functions. Punjab Univ. J. Math. 51(10), 77-91 (2019)

40. Rashid, S., Noor, M.A., Noor, A.A.O.: Some new generalizations for exponentially s-convex functions and inequalities via fractional operators. Fractal Fract. 3, Article ID 24 (2019). https://doi.org/10.3390/fractalfract3020024

41. Rashid, S., Noor, M.A., Noor, K.I., Safdar, F., Chu, Y.-M.: Hermite-Hadamard inequalities for the class of convex functions on time scale. Mathematics 7, Article ID 956 (2019). https://doi.org/10.3390/math7100956

42. Noor, M.A., Noor, K.I., Rashid, S.: Some new classes of preinvex functions and inequalities. Mathematics 7, Article ID 29 (2019). https://doi.org/10.3390/math7010029

43. Adil Khan, M., Wu, S.-H., Ullah, H., Chu, Y.-M.: Discrete majorization type inequalities for convex functions on rectangles. J. Inequal. Appl. 2019, Article ID 16 (2019)

44. Khurshid, Y., Adil Khan, M., Chu, Y.-M.: Conformable integral inequalities of the Hermite-Hadamard type in terms of GG- and GA-convexities. J. Funct. Spaces 2019, Article ID 6926107 (2019)

45. $\mathrm{Wu}, \mathrm{S}$.-H., Chu, Y.-M.: Schur $m$-power convexity of generalized geometric Bonferroni mean involving three parameters. J. Inequal. Appl. 2019, Article ID 57 (2019)

46. Zaheer Ullah, S., Adil Khan, M., Khan, Z.A., Chu, Y.-M.: Integral majorization type inequalities for the functions in the sense of strong convexity. J. Funct. Spaces 2019, Article ID 9487823 (2019)

47. Adil Khan, M., Zaheer Ullah, S., Chu, Y.-M.: The concept of coordinate strongly convex functions and related inequalities. Rev. R. Acad. Cienc. Exactas Fís. Nat., Ser. A Mat. 113(3), 2235-2251 (2019)

48. Zaheer Ullah, S., Adil Khan, M., Chu, Y.-M.: Majorization theorems for strongly convex functions. J. Inequal. Appl. 2019, Article ID 58 (2019)

49. Huang, T.-R., Tan, S.-Y., Ma, X.-Y., Chu, Y.-M.: Monotonicity properties and bounds for the complete $p$-elliptic integrals. J. Inequal. Appl. 2018, Article ID 239 (2018)

50. Wang, J.-L., Qian, W.-M., He, Z.-Y., Chu, Y.-M.: On approximating the Toader mean by other bivariate means. J. Funct. Spaces 2019, Article ID 6082413 (2019)

51. Yang, Z.-H., Qian, W.-M., Chu, Y.-M., Zhang, W.: Monotonicity rule for the quotient of two functions and its application. J. Inequal. Appl. 2017, Article ID 106 (2017)

52. Adil Khan, M., Chu, Y.-M., Khan, T.U., Khan, J.: Some new inequalities of Hermite-Hadamard type for s-convex functions with applications. Open Math. 15(1), 1414-1430 (2017)

53. Qian, W.-M., Xu, H.-Z., Chu, Y.-M.: Improvements of bounds for the Sándor-Yang means. J. Inequal. Appl. 2019, Article ID 73 (2019)

54. Wang, M.-K., Chu, Y.-M., Zhang, W.: Monotonicity and inequalities involving zero-balanced hypergeometric function. Math. Inequal. Appl. 22(2), 601-617 (2019)

55. Deng, Y., Kalsoom, H., Wu, S.: Some new quantum Hermite-Hadamard-type estimates within a class of generalized $(s, m)$-preinvex functions. Symmetry 10(11), Article ID 1283 (2019)

56. Kalsoom, H., Amer, M., Junjua, M.-ud-D., Hussain, S., Shahzadi, G.: Some (p, q)-estimates of Hermite-Hadamard-type inequalities for co-ordinated convex and quasi-convex functions. Mathematics 7(8), Article ID 683 (2019)

57. Kalsoom, H., Wu, J., Hussain, S., Latif, M.: Simpson's type inequalities for coordinated convex functions on quantum calculus. Symmetry 11(6), Article ID 768 (2019)

58. Zafar, F., Kalsoom, H., Hussain, N.: Some inequalities of Hermite-Hadamard type for n-times differentiable $(\rho, m)$-geometrically convex functions. J. Nonlinear Sci. Appl. 8(3), 201-217 (2015)

59. Kalsoom, H., Hussain, S.: Some Hermite-Hadamard type integral inequalities whose $n$-times differentiable functions are s-logarithmically convex functions. Punjab Univ. J. Math. 2019(10), 65-75 (2019) 
60. Grüss, G.: Über das Maximum des absoluten Betrages von $\frac{1}{b-a} \int_{a}^{b} f(x) g(x) d x-\frac{1}{(b-a)^{2}} \int_{a}^{b} f(x) d x \int_{a}^{b} g(x) d x$. Math. Z. 39(1), 215-226 (1935)

61. Anastassiou, G.A.: Opial type inequalities involving fractional derivatives of two functions and applications. Comput. Math. Appl. 48(10-11), 1701-1731 (2004)

62. Sulaiman, W.T.: Some new fractional integral inequalities. J. Math. Anal. 2(2), 23-28 (2011)

63. Denton, Z., Vatsala, A.S.: Fractional integral inequalities and applications. Comput. Math. Appl. 59(3), 1087-1094 (2010)

64. Mitrinović, D.S., Pečarić, J.E., Fink, A.M.: Classical and New Inequalities in Analysis. Kluwer Academic, Dordrecht (1993)

65. Yang, Z.-H., Qian, W.-M., Chu, Y.-M., Zhang, W.: On rational bounds for the gamma function. J. Inequal. Appl. 2017, Article ID 210 (2017)

66. Huang, T.-R., Han, B.-W., Ma, X.-Y., Chu, Y.-M.: Optimal bounds for the generalized Euler-Mascheroni constant. J. Inequal. Appl. 2018, Article ID 118 (2018)

67. Kilbas, S.G., Marichev, A.A.: Fractional Integrals and Derivatives, Theory and Applications. Gordon \& Breach, Yverdon (1993)

68. Kacar, E., Kacar, Z., Yildirim, H.: Integral inequalities for Riemann-Liouville fractional integrals of a function with respect to another function. Iran. J. Math. Sci. Inform. 13(1), 1-13 (2018)

69. Tariboon, J., Ntouyas, S.K., Sudsutad, W.: Some new Riemann-Liouville fractional integral inequalities. Int. J. Math. Math. Sci. 2014, Article ID 869434 (2014)

70. Kreyszig, E.: Introductory Functional Analysis with Applications. Wiley, New York (1989)

\section{Submit your manuscript to a SpringerOpen ${ }^{\circ}$} journal and benefit from:

- Convenient online submission

- Rigorous peer review

- Open access: articles freely available online

- High visibility within the field

- Retaining the copyright to your article

Submit your next manuscript at $\gg$ springeropen.com 